Chapter 30.

\title{
Lettuce, Endive, Escarole Production in Florida
}

W.M. Stall, R.N. Raid, S.E. Webb

\section{BOTANY}

\section{Nomenclature}

Family - Asteraceae (Compositae)

Lettuce - Lactuca sativa

Endive - Cichorium endiva

Escarole - Cichorium endiva

\section{Origin}

Lettuce is native to the eastern Mediterranean basin. Endive is believed to have originated in Egypt.

\section{Related Species}

Other vegetable crops in the Asteraceae family are globe artichoke, Jerusalem artichoke, radicchio, witloof chicory, salsify, cardoon, and dandelion.

\section{VARIETIES}

Crop varieties for commercial production are shown in Table 1.

\section{SEEDING AND PLANTING}

Planting dates and seeding information are given in Table 2.

\section{FERTILIZER AND LIME}

On mineral soils with subsurface or sprinkler irrigation and no mulch, broadcast all $\mathrm{P}_{2} \mathrm{O}_{5}$, micronutrients and 25 to $50 \%$ of $\mathrm{N}$ and $\mathrm{K}_{2} \mathrm{O}$ in bed. Band remaining $\mathrm{N}$ and $\mathrm{K}_{2} \mathrm{O}$ in one or two applications during middle growth period. For mulched crops with subsurface irrigation, broadcast all $\mathrm{P}_{2} \mathrm{O}_{5}$, micronutrients, and 20 to $25 \%$ of $\mathrm{N}$ and $\mathrm{K}_{2} \mathrm{O}$ in bed. Band remaining $\mathrm{N}$ and $\mathrm{K}_{2} \mathrm{O}$ in grooves in bed surface (one band for twin row beds). For mulched, drip-irrigated crops, incorporate all $\mathrm{P}_{2} \mathrm{O}_{5}$, micronutrients and up to 20 to $25 \%$ of $\mathrm{N}$ and $\mathrm{K}_{2} \mathrm{O}$ in bed. Inject remaining $\mathrm{N}$ and $\mathrm{K}_{2} \mathrm{O}$ according to schedules in circular 1181 (Fla. Coop. Ext. Serv.). Soil test and fertilizer recommendations for lettuce, endive and escarole on mineral soils are given in Table 3.

For organic soils, band all $\mathrm{P}_{2} \mathrm{O}_{5} 3$ to 4 inches beneath rows in the bed. Broadcast all $\mathrm{K}_{2} \mathrm{O}$ and micronutrients.
About $50 \mathrm{lbs} / \mathrm{A}$ of supplemental $\mathrm{N}$ might be needed during cool, winter weather, or after leaching rain. Soil test results and fertilizer recommendations for lettuce, endive, and escarole on Histosol soils are given in Table 4.

\section{PLANT TISSUE ANALYSIS}

Plant tissue analysis information for lettuce, endive and escarole is given in Table 5. The analysis was done at the 8-leaf stage, using the oldest undamaged leaf.

\section{IRRIGATION}

Crops in the lettuce group require consistent levels of water for ET (see Chapter 8, Principles and Practices of Irrigation Management for Vegetables, Tables 4 to 6) near reference demand levels (see Chapter 8, Table 3) throughout the rapid growth and final development periods. Reductions in available water during these growth periods can result in reduced leaf development. Root systems may be very shallow, thus requiring frequent, steady applications of water during low rainfall, high demand periods.

Table 1. Lettuce, endive, and escarole varieties for commercial production.

\begin{tabular}{|c|c|}
\hline Type & Variety \\
\hline \multicolumn{2}{|l|}{ Lettuce } \\
\hline \multirow[t]{3}{*}{ Crisphead (Fig. 30-1) } & Gator \\
\hline & Raleigh \\
\hline & Gulfstream \\
\hline \multirow[t]{2}{*}{ Romaine } & Terrapin \\
\hline & Snappy \\
\hline Green Leaf (Fig. 30-2) & Two Star \\
\hline \multirow[t]{2}{*}{ Red Leaf } & New Redfire \\
\hline & Vulcan \\
\hline \multirow[t]{3}{*}{ Boston } & Florida Butter Crisp (70882) \\
\hline & Ermosa \\
\hline & Margarita \\
\hline Bibb & Floribibb \\
\hline \multirow[t]{3}{*}{ Endive } & Frisan \\
\hline & Marcant \\
\hline & Salad King \\
\hline \multirow[t]{2}{*}{ Escarole (Fig. 30-3) } & Full Heart NR 65 \\
\hline & Twinkle \\
\hline
\end{tabular}




\section{WEED MANAGEMENT}

Herbicides labeled for weed control in lettuce, endive and escarole are listed in Table 6.

\section{DISEASE MANAGEMENT}

Chemicals approved for disease management are listed as follows:

Lettuce - Table 7

Endive and Escarole - Table 8.

\section{INSECT MANAGEMENT}

Insecticides approved for use on insects are listed as follows:

Lettuce - Table 9

Endive and Escarole - Table 10.

Table 2. Seeding and planting information for lettuce, endive, and escarole.

\begin{tabular}{|llllll||}
\hline Planting dates & Crisphead & Butterhead & Romaine & Endive & Escarole \\
\hline North Florida & Sept - Oct; & Sept - Oct; & Aug - Oct; & Aug - Oct; & Aug - Oct; \\
& Jan - Feb & Jan - Feb & Jan - Feb & Jan - Feb & Jan - Feb \\
Central Florida & Sept - Feb & Sept - Feb & Aug - Feb & Aug - Feb & Aug - Feb \\
South Florida & Sept - Feb & Sept - Feb & Sept - Mar & Sept - Mar & Sept - Mar \\
& & Seeding information & & & \\
& & $18-30$ & $18-30$ & $18-30$ & $18-30$ \\
Distance between rows (in) & $18-30$ & 9 & 12 & $14-16$ & $14-16$ \\
Distance between plants (in) & $8-12$ & 0.25 & 0.25 & 0.25 & 0.25 \\
Seeding depth (in) & 0.25 & $1-3$ & $2-4$ & $3-4$ & $3-4$ \\
Seed per acre (Ib) & $1-3$ & $60-80$ & $60-80$ & $60-80$ \\
Days to maturity from seed ${ }^{1}$ & $70-95$ & 38,720 & 29,040 & 24,891 & 24,891 \\
Plant populations ${ }^{2}$ (acre) & 43,560 & & & \\
\hline \hline${ }^{1}$ These crops also can be transplanted and achieve faster maturity. \\
${ }_{2}^{2}$ Populations based on closest between and within row spacing. \\
\hline
\end{tabular}

Table 3. Soil test and fertilizer recommendations for lettuce, endive, and escarole on 4-foot beds on mineral soils. ${ }^{1}$

\begin{tabular}{|c|c|c|c|c|c|c|c|c|c|c|c|}
\hline \multirow[t]{2}{*}{ Target pH } & \multirow[t]{2}{*}{$\mathrm{N} \mathrm{lb} / \mathrm{A}^{2}$} & VL & $\mathbf{L}$ & M & H & VH & VL & $\mathbf{L}$ & M & H & VH \\
\hline & & \multicolumn{4}{|c|}{$\mathrm{P}_{2} \mathrm{O}_{5}{ }^{2}$} & $(\mathrm{lb} / \mathrm{A} / \mathrm{c}$ & ason & \multicolumn{3}{|c|}{$\mathrm{K}_{2} \mathrm{O}$} & \\
\hline \multirow{2}{*}{\multicolumn{2}{|c|}{$\begin{array}{ll}\text { Crisphead lettuce } & \\
6.5 & 200 \\
\text { Leaf lettuce } & \\
6.5 & 150 \\
\text { Endive, Escarole, } & \text { Romaine }\end{array}$}} & 150 & 120 & 100 & 0 & 0 & 150 & 120 & 100 & 0 & 0 \\
\hline & & 150 & 120 & 100 & 0 & 0 & 150 & 120 & 100 & 0 & 0 \\
\hline 6.5 & 200 & 150 & 120 & 100 & 0 & 0 & 150 & 120 & 100 & 0 & 0 \\
\hline
\end{tabular}

Table 4. Soil test and fertilizer recommendations for crisphead and leaf lettuce, endive, escarole, and romaine on Histosols. Target $\mathrm{pH}=6.0$ and $\mathrm{N}$ rate $=0 \mathrm{lb} / \mathrm{A}$ for all crops.

\begin{tabular}{|c|c|c|c|c|c|c|c|c|c|c|}
\hline \multicolumn{11}{|c|}{$P$ and $K$ index and fertilizer rates } \\
\hline$P$ index & 3 & 6 & 9 & 12 & 15 & 18 & 21 & 24 & 27 & 30 \\
\hline $\mathrm{P}_{2} \mathrm{O}_{5}(\mathrm{Ib} / \mathrm{A})$ & 200 & 175 & 150 & 125 & 100 & 75 & 50 & 25 & 0 & 0 \\
\hline $\mathrm{K}$ index & 50 & 80 & 110 & 140 & 170 & 200 & & & & \\
\hline $\mathrm{K}_{2} \mathrm{O}(\mathrm{Ib} / \mathrm{A})$ & 200 & 140 & 80 & 50 & 0 & 0 & & & & \\
\hline
\end{tabular}


Table 5. Plant tissue analysis at 8-leaf stage for lettuce, endive, escarole, and romaine. Dry wt. basis.

\begin{tabular}{|c|c|c|c|c|c|c|c|c|c|c|c|c|}
\hline \multirow[b]{2}{*}{ Status } & $\mathbf{N}$ & $\mathbf{P}$ & K & $\mathrm{Ca}$ & $\mathrm{Mg}$ & $S$ & $\mathrm{Fe}$ & Mn & $\mathrm{Zn}$ & B & $\mathrm{Cu}$ & Mo \\
\hline & \multicolumn{6}{|c|}{ Percent } & \multicolumn{6}{|c|}{ Parts per million } \\
\hline \multicolumn{13}{|l|}{ Crisphead } \\
\hline Deficient & $<4.0$ & 0.4 & 5.0 & 0.8 & 0.3 & 0.3 & 50 & 20 & 25 & 15 & 5 & 0.1 \\
\hline Adequate range & $4.0-5.0$ & $0.4-0.6$ & $5.0-7.0$ & $0.8-2.0$ & $0.3-0.5$ & $0.3-0.8$ & $50-150$ & $20-40$ & $25-50$ & $15-30$ & $5-10$ & $0.1-0.4$ \\
\hline High & $>5$ & 0.6 & 7.0 & 2.0 & 0.5 & 0.8 & 150 & 40 & 50 & 30 & 10 & 0.4 \\
\hline \multicolumn{13}{|l|}{ Endive } \\
\hline Deficient & $<4.5$ & 0.45 & 4.5 & 0.8 & 0.25 & 0.3 & 50 & 15 & 30 & 25 & 5 & 0.1 \\
\hline Adequate range & $4.5-6.0$ & $0.45-0.8$ & $4.5-6.0$ & $0.8-4.0$ & $0.25-0.60$ & $0.3-0.8$ & $50-150$ & $15-25$ & $30-50$ & $25-35$ & $5-10$ & $0.1-0.4$ \\
\hline High & $>6.0$ & 0.8 & 6.0 & 4.0 & 0.6 & 0.8 & 150 & 25 & 50 & 35 & 10 & 0.4 \\
\hline \multicolumn{13}{|l|}{ Escarole } \\
\hline Deficient & $<4.2$ & 0.45 & 5.7 & 0.8 & 0.25 & 0.3 & 50 & 15 & 30 & 20 & 4 & 0.1 \\
\hline Adequate range & $4.2-5.0$ & $0.45-0.6$ & $5.7-6.5$ & $0.8-2.2$ & $0.25-0.35$ & $0.3-0.8$ & $50-150$ & $15-25$ & $30-50$ & $20-30$ & $4-6$ & $0.1-0.4$ \\
\hline High & $>5.0$ & 0.6 & 6.5 & 2.2 & 0.35 & 0.8 & 150 & 25 & 50 & 30 & 6 & 0.4 \\
\hline \multicolumn{13}{|l|}{ Romaine } \\
\hline Deficient & $<5.0$ & 0.35 & 5.0 & 0.8 & 0.25 & 0.3 & 50 & 15 & 20 & 30 & 5 & 0.1 \\
\hline Adequate range & $5.0-6.0$ & $0.35-0.8$ & $5.0-6.0$ & $0.8-3.0$ & $0.25-0.35$ & $50.3-0.8$ & $50-150$ & $15-25$ & $20-50$ & $30-45$ & $5-10$ & $0.1-0.4$ \\
\hline High & $>6.0$ & 0.8 & 6.0 & 3.0 & 0.35 & 0.8 & 150 & 25 & 50 & 45 & 10 & 0.4 \\
\hline
\end{tabular}

Table 6. Chemical weed controls: lettuce, endive and escarole.

\begin{tabular}{||lllll|}
\hline & & Time of & \multicolumn{2}{c|}{ Rate (Ibs. Al./Acre) } \\
\cline { 3 - 5 } Herbicide & Labeled crops & application to crop & Mineral & Muck \\
\hline \hline Benefin (Balan) & $\begin{array}{l}\text { Lettuce } \\
\text { (direct seeded) }\end{array}$ & Preplant incorporated & $1.12-1.5$ & --- \\
\end{tabular}

Remarks: Controls germinating annuals. Incorporate 2 to 3 inches within 8 hours. Not recommended for organic soils.

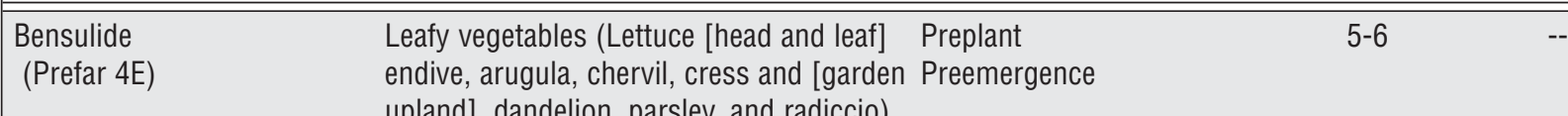

upland], dandelion, parsley, and radiccio)

Remarks: Preplant incorporate using power driven rotary cultivations or apply preemergence and incorporate with irrigation. Use preemergence only with lettuce to be irrigated up. Controls many grasses. Provides fair to good control of lambsquarter, purslane, and amaranths.

\begin{tabular}{lllll}
\hline \hline Carfentrazone & Leafy vegetables & Preplant & 0.031 & 0.031 \\
(Aim) & (All) & Directed-hooded & & \\
& & Row-middles & &
\end{tabular}

Remarks: Aim may be applied as a preplant burndown treatment and/or as a post-directed hooded application to row middles for the burndown of emerged broadleaf weeds. May be tank mixed with other registered herbicides. may be applied at up to 2 oz ( $0.031 \mathrm{lb}$ ai). Use a quality spray adjuvant such as crop oil concentrate (coc) or non-ionic surfactant at recommended rates.

\begin{tabular}{lllll}
\hline Fluazifop (Fusilade DX) & Endive & Postemergence & 0.188 & 0.188
\end{tabular}

Remarks: Controls actively growing grass weeds. A total of $48 \mathrm{oz}$. may be applied per season. Do not apply within 28 days of harvest for endive. Use a crop oil concentrate at $0.5-1 \% \mathrm{v} / \mathrm{v}$ or a non-ionic surfactant at $0.25-0.5 \% \mathrm{v} / \mathrm{v}$ in spray mixture. Consult the label for specific rates and grass growth stages for best control.

\begin{tabular}{llll}
\hline \hline Glyphosate & Leafy vegetables & Chemical fallow & $0.3-1.0$ \\
(Roundup, Durango) & & Preplant, pre emergence, & \\
Touchdown, Glyphomax) & & Pre transplant & \\
\hline
\end{tabular}

Remarks: Roundup, Glyphomax and Touchdown have several formulations. Check the label of each for specific labeling directions.

\begin{tabular}{lllr}
\hline \hline Imazethapyr & Lettuce, Endive, Escarole & Preemergence & $0.015-0.03$ \\
(Pursuit) & & Postemergence & ---- \\
\hline
\end{tabular}

Remarks: Third Party Registration (TPR, Inc.) only. Apply with ground equipment only at broadcast rates of 1 to 2 ounces material per acre preemergence and/or postemergence after 3-4 true leaf stage. Do not apply more than 2 applications per crop. A maximum of 4 ounces of product may be used per crop season and 6 ounces of product per acre per calendar year. Should be applied in 20 or more gallons of water per acre. Do not apply within 30 days of harvest. Potential for rotational crop damage is highly variable. Do not plant other crops within 45 days of application.

Use of Pursuit on lettuce, endive, or escarole, without having a signed authorization and waiver and limitation of liability agreement is a misuse of the product. 
Table 6. Continued.

\begin{tabular}{|c|c|c|c|c|}
\hline \multirow[b]{2}{*}{ Herbicide } & \multirow[b]{2}{*}{ Labeled crops } & \multirow{2}{*}{$\begin{array}{l}\text { Time of } \\
\text { application to crop }\end{array}$} & \multicolumn{2}{|c|}{ Rate (Ibs. Al./Acre) } \\
\hline & & & Mineral & Muck \\
\hline $\begin{array}{l}\text { Paraquat } \\
\text { (Gramoxone Intron) } \\
\text { (Firestorm) } \\
\text { Remarks: Controls emergec }\end{array}$ & weeds. Apply prior to, during or & t seeding, but before lettuce en & $0.63-0.94$ & $0.63-0.94$ \\
\hline $\begin{array}{l}\text { Paraquat } \\
\text { (Gramoxone Intron) } \\
\text { Remarks: A Special Local N } \\
\text { when weeds are } 1 \text { to } 6 \text { inche }\end{array}$ & $\begin{array}{l}\text { Lettuce } \\
\text { eeds } 24(\mathrm{c}) \text { Label for Florida Only } \\
\text { s tall, using } 1.5 \text { pts. per acre. Us } \\
\end{array}$ & $\begin{array}{l}\text { Postemergence as a directed/ } \\
\text { shielded spray } \\
\text { emerged weeds. Apply as a direc } \\
\text { ic spreader. }\end{array}$ & $\begin{array}{l}0.47 \\
\text { /shielded sp }\end{array}$ & $\begin{array}{l}0.47 \\
\text { between rows }\end{array}$ \\
\hline \multicolumn{4}{|c|}{$\begin{array}{l}\text { Remarks: Product is a contact non-selective, foliar applied herbicide. There is no residual activity. May be tank mixed with soil residual } \\
\text { compounds. Consult the label for rates and other information. }\end{array}$} & $\begin{array}{l}3-10 \% v / v \\
\text { soil residual }\end{array}$ \\
\hline \multicolumn{5}{|c|}{$\begin{array}{l}\text { Remarks: Controls germinating annuals. Overhead-irrigate briefly or incorporate } 2 \text { to } 3 \text { inches. Note precautions of planting non-regis- } \\
\text { tered or sensitive crops after application. Not recommended for organic soils. }\end{array}$} \\
\hline $\begin{array}{l}\text { Sethoxydim (Poast) } \\
\text { Remarks: Controls actively } \\
\text { days of harvest for head lett } \\
\text { oil concentrate per acre. Uns } \\
\text { and up to } 0.28 \mathrm{lb} \text { ai. (1.5 pt } \\
\text { best control. } \\
\end{array}$ & $\begin{array}{l}\text { Lettuce: Head, Leaf, Endive } \\
\text { growing grass weeds. A total of } \\
\text { Ice and } 15 \text { days of harvest for lea } \\
\text { atisfactory results may occur if a }\end{array}$ & $\begin{array}{l}\text { Postemergence } \\
\text { ct per acre may be applied in } \\
\text { id spinach. Apply in } 5 \text { to } 20 \text { ga } \\
\text { asses under stress. Use } 0.188\end{array}$ & $\begin{array}{l}0.188-0.2 \\
\text { season. Do n } \\
\text { f water addin } \\
\text { ai. (1 pt.) to }\end{array}$ & $\begin{array}{l}0.188-0.28 \\
\text { apply within } 30 \\
\text { pts. of crop } \\
\text { dling grasses } \\
\text { th stage for }\end{array}$ \\
\hline $\begin{array}{l}\text { Trifluralin } \\
\text { (Treflan EC, Treflan 5) } \\
\text { Treflan MFT, Trifluralin 4EC } \\
\text { Remarks: Apply as a prepla }\end{array}$ & $\begin{array}{l}\text { Endive, Escarole, Radicchio } \\
\text { nt incorporated treatment to min }\end{array}$ & $\begin{array}{l}\text { Preplant } \\
\text { Incorporate } \\
\text { nly. Consult label for applicat }\end{array}$ & 0.5 & 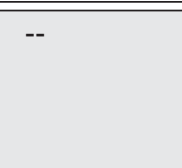 \\
\hline
\end{tabular}

Table 7. Disease management for lettuce.

\begin{tabular}{|c|c|c|c|c|c|c|}
\hline Chemical (a.i.) & $\begin{array}{l}\text { FRAC } \\
\text { Group1 }\end{array}$ & $\frac{\text { Maximum }}{\text { Application }}$ & $\frac{\text { Rate/Acre/ }}{\text { Season }}$ & $\begin{array}{l}\text { Min. Days } \\
\text { to Harvest }\end{array}$ & $\begin{array}{l}\text { Pertinent Diseases } \\
\text { or Pathogens }\end{array}$ & Remarks $^{2}$ \\
\hline $\begin{array}{l}\text { Acrobat 50WP } \\
\text { (Dimethomorph) }\end{array}$ & 40 & $6.40 z$ & $320 z$ & 0 & Downy mildew & $\begin{array}{l}\text { Do not exceed } 2 \text { sequential and } 5 \\
\text { total applications. Note rotational } \\
\text { restrictions (see label) }\end{array}$ \\
\hline $\begin{array}{l}\text { Aliette 80WDG } \\
\text { (Fosetyl Al) }\end{array}$ & 33 & $5 \mathrm{lb}$ & $35 \mathrm{lb}$ & 3 & Downy mildew & $\begin{array}{l}\text { Do not mix with copper fungi- } \\
\text { cides. }\end{array}$ \\
\hline $\begin{array}{l}\text { Amistar 80DF } \\
\text { (Azoxystrobin) }\end{array}$ & 11 & $50 z$ & $200 z$ & 0 & $\begin{array}{l}\text { Alternaria leaf spot, } \\
\text { Cercospora leaf } \\
\text { spot, Downy mildew, } \\
\text { Powdery mildew, } \\
\text { Septoria leaf spot }\end{array}$ & $\begin{array}{l}\text { Do not exceed } 1 \text { sequential and } 4 \\
\text { total applications of Amistar or } \\
\text { other Qol fungicides. }\end{array}$ \\
\hline $\begin{array}{l}\text { Apron XL LS } \\
\text { (Mefenoxam) }\end{array}$ & 4 & $\begin{array}{l}0.64 \mathrm{fl} .0 \mathrm{0z} . / \\
100 \mathrm{lb} \text { seed }\end{array}$ & & & Pythium seedling blight & Seed treatment only \\
\hline $\begin{array}{l}\text { Armicarb } 100 \\
\text { (Potassium bicar- } \\
\text { bonate) }\end{array}$ & & $5 \mathrm{lb}$ & & 0 & $\begin{array}{l}\text { Powdery mildew, } \\
\text { Alternaria leaf spot, } \\
\text { Septoria leaf spot, Gray } \\
\text { mold }\end{array}$ & $\begin{array}{l}\text { Do not exceed } 5 \text { lbs per } 100 \\
\text { gallons of water. Spray solution } \\
\text { within } 12 \text { hours of preparation. }\end{array}$ \\
\hline $\begin{array}{l}\text { Basic Copper } 53 \\
\text { (Basic copper sul- } \\
\text { fate) }\end{array}$ & M1 & $3 \mathrm{lb}$ & & & $\begin{array}{l}\text { Downy mildew } \\
\text { Bacterial leaf spot }\end{array}$ & \\
\hline
\end{tabular}


Table 7. Continued.

\begin{tabular}{|c|c|c|c|c|c|c|}
\hline Chemical (a.i.) & $\begin{array}{l}\text { FRAC } \\
\text { Group1 }\end{array}$ & $\frac{\text { Maximum }}{\text { Application }}$ & $\frac{\text { Rate/Acre/ }}{\text { Season }}$ & $\begin{array}{l}\text { Min. Days } \\
\text { to Harvest }\end{array}$ & $\begin{array}{l}\text { Pertinent Diseases } \\
\text { or Pathogens }\end{array}$ & Remarks $^{2}$ \\
\hline $\begin{array}{l}\text { Botran 75W } \\
\text { (Dicloran) }\end{array}$ & 14 & $5.33 \mathrm{lb}$ & $5.33 \mathrm{lb}$ & 14 & $\begin{array}{l}\text { Botrytis rot } \\
\text { Sclerotinia diseases }\end{array}$ & $\begin{array}{l}\text { Some leaf bronzing can occur but } \\
\text { effect is temporary. See label for } \\
\text { specifics on application timing } \\
\text { and placement. }\end{array}$ \\
\hline $\begin{array}{l}\text { Cabrio EG } \\
\text { (Pyraclostrobin) }\end{array}$ & 11 & $160 z$ & $640 z$ & 0 & $\begin{array}{l}\text { Alternaria leaf spot, } \\
\text { Cercospora leaf } \\
\text { spot, Downy mildew, } \\
\text { Powdery mildew, } \\
\text { Septoria leaf spot }\end{array}$ & $\begin{array}{l}\text { Do not exceed } 2 \text { sequential and } \\
4 \text { total applications of Cabrio or } \\
\text { other Qol fungicides. }\end{array}$ \\
\hline $\begin{array}{l}\text { Champ } 77 \text { WP } \\
\text { (Copper hydroxide) }\end{array}$ & M1 & $2 \mathrm{lb}$ & & 2 & $\begin{array}{l}\text { Downy mildew } \\
\text { Bacterial leaf spot }\end{array}$ & \\
\hline $\begin{array}{l}\text { Champ DP Dry Prill } \\
\text { (Copper hydroxide) }\end{array}$ & M1 & $1.33 \mathrm{lb}$ & & 1 & $\begin{array}{l}\text { Downy mildew } \\
\text { Bacterial leaf spot }\end{array}$ & \\
\hline $\begin{array}{l}\text { Champ Formula 2 F } \\
\text { (Copper hydroxide) }\end{array}$ & M1 & $1.33 \mathrm{pt}$ & & 1 & $\begin{array}{l}\text { Downy mildew } \\
\text { Bacterial leaf spot }\end{array}$ & \\
\hline $\begin{array}{l}\text { Contans WG } \\
\text { (Coniothryium mini- } \\
\text { tans) }\end{array}$ & & $6 \mathrm{lbs}$ & & & Lettuce drop & $\begin{array}{l}\text { Apply to soil surface and incor- } \\
\text { porate prior to, at planting, or at } \\
\text { transplanting. }\end{array}$ \\
\hline $\begin{array}{l}\text { Dusting Sulfur - IAP } \\
\text { (Sulfur) }\end{array}$ & M2 & $15 \mathrm{lb}$ & & & Powdery mildew & $\begin{array}{l}\text { Do not apply during periods of } \\
\text { warm weather. Do not apply } \\
\text { within } 2 \text { weeks of an oil spray. }\end{array}$ \\
\hline $\begin{array}{l}\text { Endura 70WG } \\
\text { (Boscalid) }\end{array}$ & 7 & $110 z$ & $220 z$ & 14 & $\begin{array}{l}\text { Lettuce drop, } \\
\text { Rhizoctonia, Botrytis } \\
\text { rot }\end{array}$ & $\begin{array}{l}\text { Do not exceed } 2 \text { applications } \\
\text { per crop. See label for timing of } \\
\text { applications on direct-seeded and } \\
\text { transplanted lettuce. }\end{array}$ \\
\hline $\begin{array}{l}\text { Fosphite } \\
\text { (Potassium phos- } \\
\text { phite) }\end{array}$ & 33 & $3 \mathrm{qt}$ & $18 \mathrm{qt}$ & & $\begin{array}{l}\text { Downy mildew, } \\
\text { Pythium, Rhizoctonia, } \\
\text { Fusarium }\end{array}$ & $\begin{array}{l}\text { Do not exceed } 6 \text { applications per } \\
\text { crop. Caution should be used } \\
\text { when applying in a management } \\
\text { program including copper fun- } \\
\text { gicides. }\end{array}$ \\
\hline $\begin{array}{l}\text { Helena Prophyt } \\
\text { (Potassium phos- } \\
\text { phite) }\end{array}$ & 33 & $4 \mathrm{pt}$ & $18 \mathrm{qt}$ & 0 & $\begin{array}{l}\text { Downy mildew, } \\
\text { Pythium }\end{array}$ & $\begin{array}{l}\text { Apply in a minimum spray vol- } \\
\text { ume of } 30 \mathrm{GPA} \text {. Caution should } \\
\text { be used when applying in a } \\
\text { management program including } \\
\text { copper fungicides. }\end{array}$ \\
\hline $\begin{array}{l}\text { Iprodione 4L AG } \\
\text { (Iprodione) }\end{array}$ & 2 & $2 \mathrm{pt}$ & $6 \mathrm{pt}$ & 14 & $\begin{array}{l}\text { Rhizoctonia bottom rot, } \\
\text { Sclerotinia drop }\end{array}$ & $\begin{array}{l}\text { Limit is } 3 \text { applications per crop. } \\
\text { See label for timing and place- } \\
\text { ment. }\end{array}$ \\
\hline $\begin{array}{l}\text { Kaligreen } \\
\text { (Potassium bicar- } \\
\text { bonate) }\end{array}$ & & $3 \mathrm{lb}$ & & 1 & Powdery mildew & $\begin{array}{l}\text { Apply in a minimum spray vol- } \\
\text { ume of } 25 \mathrm{GPA} \text {. }\end{array}$ \\
\hline $\begin{array}{l}\text { Kumulus DF } \\
\text { (Sulfur) }\end{array}$ & M2 & $6 \mathrm{lb}$ & & & Powdery mildew & $\begin{array}{l}\text { Avoid application when tempera- } \\
\text { tures exceed } 90 \mathrm{~F} \text {, as phytotoxic- } \\
\text { ity may result. Apply when dis- } \\
\text { ease first appears and at } 14 \text {-day } \\
\text { intervals or as needed. }\end{array}$ \\
\hline $\begin{array}{l}\text { Maneb 80WP } \\
\text { (Maneb) }\end{array}$ & M3 & $2 \mathrm{lb}$ & $12 \mathrm{lb}$ & 10 & Downy mildew & \\
\hline $\begin{array}{l}\text { Maneb 75DF } \\
\text { (Maneb) }\end{array}$ & M3 & $2 \mathrm{lb}$ & $12.8 \mathrm{lb}$ & 10 & Downy mildew & \\
\hline $\begin{array}{l}\text { Manex 4F } \\
\text { (Maneb) }\end{array}$ & M3 & $1.6 \mathrm{qt}$ & $9.6 \mathrm{qt}$ & 10 & Downy mildew & \\
\hline $\begin{array}{l}\text { Maxim 4FS } \\
\text { (Fludioxonil) }\end{array}$ & 12 & $\begin{array}{l}0.16 \mathrm{fl} \mathrm{oz/} \\
100 \mathrm{lbs} \text { of } \\
\text { seed }\end{array}$ & & & $\begin{array}{l}\text { Various seedling dis- } \\
\text { eases }\end{array}$ & Seed treatment only. \\
\hline
\end{tabular}


Table 7. Continued.

\begin{tabular}{|c|c|c|c|c|c|c|}
\hline Chemical (a.i.) & $\begin{array}{l}\text { FRAC } \\
\text { Group1 }\end{array}$ & $\frac{\text { Maximum }}{\text { Application }}$ & $\frac{\text { Rate/Acre/ }}{\text { Season }}$ & $\begin{array}{l}\text { Min. Days } \\
\text { to Harvest }\end{array}$ & $\begin{array}{l}\text { Pertinent Diseases } \\
\text { or Pathogens }\end{array}$ & Remarks $^{2}$ \\
\hline $\begin{array}{l}\text { Micro Sulf } \\
\text { (Sulfur) }\end{array}$ & M2 & $6 \mathrm{lb}$ & & & Powdery mildew & $\begin{array}{l}\text { Do not apply during periods of } \\
\text { warm weather. Do not apply } \\
\text { within } 2 \text { weeks of an oil spray. }\end{array}$ \\
\hline $\begin{array}{l}\text { Micronized Gold } \\
\text { (Sulfur) }\end{array}$ & M2 & $5 \mathrm{lb}$ & & & Powdery mildew, Rust & $\begin{array}{l}\text { Do not apply during periods of } \\
\text { warm weather. Do not apply } \\
\text { within } 2 \text { weeks of an oil spray. }\end{array}$ \\
\hline $\begin{array}{l}\text { Microthiol Disperss } \\
\text { (Sulfur) }\end{array}$ & M2 & $10 \mathrm{lb}$ & & & Powdery mildew & $\begin{array}{l}\text { Do not apply during periods of } \\
\text { warm weather. Do not apply } \\
\text { within } 2 \text { weeks of an oil spray. }\end{array}$ \\
\hline $\begin{array}{l}\text { Milstop } \\
\text { (Potassium bicar- } \\
\text { bonate) }\end{array}$ & & $5 \mathrm{lb}$ & & & $\begin{array}{l}\text { Downy mildew } \\
\text { Powdery mildew }\end{array}$ & $\begin{array}{l}\text { Do not store unused spray solu- } \\
\text { tion. Apply in sufficient volume to } \\
\text { get adequate crop coverage. }\end{array}$ \\
\hline $\begin{array}{l}\text { Nordox } \\
\text { (Cuprous oxide) }\end{array}$ & & $2 \mathrm{lb}$ & & & $\begin{array}{l}\text { Downy mildew } \\
\text { Bacterial leaf spot }\end{array}$ & \\
\hline $\begin{array}{l}\text { Nordox 75WG } \\
\text { (Cuprous oxide) }\end{array}$ & & $2.5 \mathrm{lb}$ & & & $\begin{array}{l}\text { Downy mildew } \\
\text { Bacterial leaf spot }\end{array}$ & \\
\hline $\begin{array}{l}\text { Nu-Cop 3L } \\
\text { (Copper hydroxide) }\end{array}$ & M1 & $2.66 \mathrm{pt}$ & & & $\begin{array}{l}\text { Downy mildew } \\
\text { Bacterial leaf spot }\end{array}$ & \\
\hline $\begin{array}{l}\text { Nu-Cop 50DF } \\
\text { (Copper hydroxide) }\end{array}$ & M1 & $2.0 \mathrm{lb}$ & & & $\begin{array}{l}\text { Downy mildew } \\
\text { Bacterial leaf spot }\end{array}$ & \\
\hline $\begin{array}{l}\text { Nu-Cop 50WP } \\
\text { (Copper hydroxide) }\end{array}$ & M1 & $2.0 \mathrm{lb}$ & & & $\begin{array}{l}\text { Downy mildew } \\
\text { Bacterial leaf spot }\end{array}$ & \\
\hline $\begin{array}{l}\text { Oxidate } \\
\text { (Hydrogen dioxide) }\end{array}$ & & $128 \mathrm{fl} \mathrm{oz}$ & & 0 & & \\
\hline $\begin{array}{l}\text { Phostral } \\
\text { (Potassium phospite) }\end{array}$ & 33 & $5 \mathrm{pt}$ & $35 \mathrm{pt}$ & 0 & Downy mildew & $\begin{array}{l}\text { Do not exceed } 7 \text { applications per } \\
\text { season. See label regarding cau- } \\
\text { tions regarding conditions during } \\
\text { application to avoid possible } \\
\text { phytotoxicity. }\end{array}$ \\
\hline $\begin{array}{l}\text { PlantShield HC } \\
\text { (Tricoderma harzia- } \\
\text { num) }\end{array}$ & & $50 z$ & & & & $\begin{array}{l}\text { Foliar and root fungicide. Use as } \\
\text { a drench. (see label for restric- } \\
\text { tions). }\end{array}$ \\
\hline $\begin{array}{l}\text { Previcur Flex } \\
\text { (Propamacarb) }\end{array}$ & U & $2 \mathrm{pt}$ & $8 \mathrm{pt}$ & 2 & Downy mildew & $\begin{array}{l}\text { Avoid sequential applications } \\
\text { for same pathogen. Use in IPM } \\
\text { program with fungicides of dis- } \\
\text { similar group. Do not rotate to } \\
\text { root or leafy vegetables within } 30 \\
\text { days of harvest }\end{array}$ \\
\hline $\begin{array}{l}\text { Quadris } \\
\text { (Azoxystrobin) }\end{array}$ & 11 & $15.4 \mathrm{fl} \mathrm{oz}$ & $2.88 \mathrm{qt}$ & 0 & $\begin{array}{l}\text { Alternaria leaf spot, } \\
\text { Cercospora leaf } \\
\text { spot, Downy mildew, } \\
\text { Powdery mildew, } \\
\text { Septoria leaf spot }\end{array}$ & $\begin{array}{l}\text { Do not exceed } 1 \text { sequential and } \\
4 \text { total applications of Quadris } \\
\text { or other Qol fungicides. See } \\
\text { label for cautions regarding tank } \\
\text { mixtures to avoid possible phy- } \\
\text { toxicity. }\end{array}$ \\
\hline $\begin{array}{l}\text { Reason 500SC } \\
\text { (Fenamidone) }\end{array}$ & 11 & $8.2 \mathrm{fl} \mathrm{oz}$ & $24.6 \mathrm{fl} \mathrm{oz}$ & 2 & Downy mildew & $\begin{array}{l}\text { Do not exceed } 1 \text { sequential appli- } \\
\text { cation of Reason or other Qol } \\
\text { fungicides before alternating to } \\
\text { a different fungicide group. See } \\
\text { label for crop rotation restric- } \\
\text { tions. }\end{array}$ \\
\hline $\begin{array}{l}\text { Rhapsody } \\
\text { (Bacillus subtilis) }\end{array}$ & & 6 qt & & 0 & $\begin{array}{l}\text { Downy mildew } \\
\text { Powdery mildew } \\
\text { Sclerotinia drop }\end{array}$ & $\begin{array}{l}\text { For suppression or use as a } \\
\text { preventative in a program with } \\
\text { other registered fungicides. For } \\
\text { Sclerotinia, apply as a banded } \\
\text { spray (see label for placement } \\
\text { and timings). }\end{array}$ \\
\hline
\end{tabular}


Table 7. Continued.

\begin{tabular}{|c|c|c|c|c|c|c|}
\hline Chemical (a.i.) & $\begin{array}{l}\text { FRAC } \\
\text { Group1 }\end{array}$ & $\frac{\text { Maximum }}{\text { Application }}$ & $\begin{array}{l}\text { Rate/Acre/ } \\
\text { Season }\end{array}$ & $\begin{array}{l}\text { Min. Days } \\
\text { to Harvest }\end{array}$ & $\begin{array}{l}\text { Pertinent Diseases } \\
\text { or Pathogens }\end{array}$ & Remarks $^{2}$ \\
\hline $\begin{array}{l}\text { Ridomil Gold EC } \\
\text { (Mefenoxam) }\end{array}$ & 4 & 2 pts & & & $\begin{array}{l}\text { Pythium seedling dis- } \\
\text { eases }\end{array}$ & $\begin{array}{l}\text { Apply at seeding in a } 7-12 \text { " band } \\
\text { on soil over seed furrow }\end{array}$ \\
\hline $\begin{array}{l}\text { Ridomil Gold GR } \\
\text { (Mefenoxam) }\end{array}$ & 4 & $40 \mathrm{lb}$ & & & $\begin{array}{l}\text { Pythium seedling dis- } \\
\text { eases }\end{array}$ & $\begin{array}{l}\text { See label for specifics regarding } \\
\text { preplant incorporated applica- } \\
\text { tions and surface applications at } \\
\text { the time of planting. }\end{array}$ \\
\hline $\begin{array}{l}\text { RootShield Granules } \\
\text { (Tricoderma harzia- } \\
\text { num) }\end{array}$ & & $12 \mathrm{lb}$ & & & & Apply in-furrow. \\
\hline $\begin{array}{l}\text { Rovral 4F } \\
\text { (Iprodione) }\end{array}$ & 2 & $2 \mathrm{pt}$ & $6 \mathrm{pt}$ & 14 & $\begin{array}{l}\text { Rhizoctonia bottom rot } \\
\text { Sclerotinia drop }\end{array}$ & $\begin{array}{l}\text { Limit is } 3 \text { applications per crop. } \\
\text { See label for details on applica- } \\
\text { tion timing and placement. }\end{array}$ \\
\hline $\begin{array}{l}\text { Serenade Max } \\
\text { (Bacillus subtilis) }\end{array}$ & & $3 \mathrm{lb}$ & & 0 & $\begin{array}{l}\text { Downy mildew } \\
\text { Powdery mildew } \\
\text { Sclerotinia drop }\end{array}$ & $\begin{array}{l}\text { For suppression or use as a } \\
\text { preventative in a program with } \\
\text { other registered fungicides. For } \\
\text { Sclerotinia, apply as a banded } \\
\text { spray (see label for placement } \\
\text { and timings). }\end{array}$ \\
\hline $\begin{array}{l}\text { Sonata } \\
\text { (Bacillus pumilus) }\end{array}$ & & $4 \mathrm{qt}$ & & 0 & $\begin{array}{l}\text { Downy mildew } \\
\text { Powdery mildew }\end{array}$ & $\begin{array}{l}\text { For suppression or use as a pre- } \\
\text { ventative in a program with other } \\
\text { registered fungicides. }\end{array}$ \\
\hline $\begin{array}{l}\text { Sporan } \\
\text { (Clove, Rosemary, } \\
\text { and Thyme Oil) }\end{array}$ & & $1.5 \mathrm{qt}$ & & 0 & $\begin{array}{l}\text { Botrytis gray mold } \\
\text { Powdery mildew }\end{array}$ & $\begin{array}{l}\text { Sporan is a concentrated oil- } \\
\text { based product. It requires the } \\
\text { use of an approved adjuvant to } \\
\text { improve spreading and sticking. } \\
\text { OMRI listed. }\end{array}$ \\
\hline $\begin{array}{l}\text { Stretch } \\
\text { (Copper hydroxide) }\end{array}$ & M1 & $4 \mathrm{pt}$ & & & $\begin{array}{l}\text { Downy mildew } \\
\text { Bacterial leaf spot }\end{array}$ & \\
\hline $\begin{array}{l}\text { Sulfur 90W } \\
\text { (Sulfur) }\end{array}$ & M2 & $6 \mathrm{lb}$ & & & Powdery mildew & $\begin{array}{l}\text { Do not apply during periods of } \\
\text { warm weather. Do not apply } \\
\text { within } 2 \text { weeks of an oil spray. }\end{array}$ \\
\hline $\begin{array}{l}\text { Tanos } \\
\text { (Cymoxanil, } \\
\text { Famoxadone) }\end{array}$ & 27,11 & $80 z$ & $240 z$ & 3 & Downy mildew & $\begin{array}{l}\text { Do not exceed } 1 \text { sequential and } \\
3 \text { total applications of Tanos or } \\
\text { other Qol fungicides. }\end{array}$ \\
\hline $\begin{array}{l}\text { Telone EC } \\
\text { (1,3, dichloropro- } \\
\text { pene) }\end{array}$ & & $18 \mathrm{gal}$ & & & $\begin{array}{l}\text { Nematode and soil- } \\
\text { borne diseases }\end{array}$ & $\begin{array}{l}\text { Apply as a soil fumigant. } \\
\text { Restricted use pesticide. See } \\
\text { label regarding specific applica- } \\
\text { tion instructions. }\end{array}$ \\
\hline $\begin{array}{l}\text { Tenn-Cop 5E } \\
\text { (Copper tallate) }\end{array}$ & M1 & $3 \mathrm{pt}$ & & & $\begin{array}{l}\text { Downy mildew } \\
\text { Bacterial soft rot }\end{array}$ & $\begin{array}{l}\text { Full season use at high rate may } \\
\text { result in marginal yellowing. Do } \\
\text { not apply in a solution with a pH } \\
\text { of less than } 6.5 \text {. }\end{array}$ \\
\hline $\begin{array}{l}\text { Topaz } \\
\text { (Potassium phos- } \\
\text { phite) }\end{array}$ & 33 & $3 \mathrm{qt}$ & $18 \mathrm{qt}$ & 0 & Downy mildew & \\
\hline $\begin{array}{l}\text { Trilogy } \\
\text { (Neem Oil) }\end{array}$ & & $2 \mathrm{gal}$ & & & & $\begin{array}{l}\text { Apply at a rate of } 0.5 \%-1.0 \% \\
\text { in } 25 \text { to } 100 \text { gallons of water per } \\
\text { acre or at } 2 \text { pt in a minimum of } 5 \\
\text { GPA for low volume applications. }\end{array}$ \\
\hline $\begin{array}{l}\text { Ultra Flourish } \\
\text { (Mefenoxam) }\end{array}$ & 4 & $4 \mathrm{pt}$ & & & $\begin{array}{l}\text { Pythium seedling dis- } \\
\text { ease }\end{array}$ & $\begin{array}{l}\text { See label for specifics regarding } \\
\text { preplant incorporated applica- } \\
\text { tions and surface applications at } \\
\text { the time of planting. }\end{array}$ \\
\hline
\end{tabular}


Table 8. Disease management for endive and escarole.

\begin{tabular}{|c|c|c|c|c|c|}
\hline \multirow[b]{2}{*}{ Chemical } & \multicolumn{2}{|c|}{ Maximum Rate/Acre/ } & \multirow{2}{*}{$\begin{array}{l}\text { Minimum Days } \\
\text { to Harvest }\end{array}$} & \multirow[b]{2}{*}{ Pertinent Diseases } & \multirow[b]{2}{*}{ Select Remarks } \\
\hline & Application & Crop & & & \\
\hline Maneb 75DF & $2 \mathrm{lbs}$ & $12.8 \mathrm{lbs}$ & & $\begin{array}{l}\text { Downy mildew, } \\
\text { Alternaria leaf spot }\end{array}$ & \\
\hline Maneb 80WP & $2 \mathrm{lbs}$ & $12 \mathrm{lbs}$ & 10 & $\begin{array}{l}\text { Downy mildew, } \\
\text { Alternaria leaf spot }\end{array}$ & \\
\hline Manex 4F & 1.6 qts & 9.6 qts & 10 & $\begin{array}{l}\text { Downy mildew, } \\
\text { Alternaria leaf spot }\end{array}$ & \\
\hline Ridomil Gold 4EC & $2 \mathrm{pts} / \mathrm{trt} \mathrm{A}$ & & 10 & Pythium blight & $\begin{array}{l}\text { Apply at seeding in a 7-12" } \\
\text { band on soil after seeding. }\end{array}$ \\
\hline Botran 75W & $2 / 3 \mathrm{lbs}$ & $5 \frac{1}{3} \mathrm{lbs}$ & 14 & $\begin{array}{l}\text { Botrytis } \\
\text { Sclerotinia }\end{array}$ & $\begin{array}{l}\text { Some leaf bronzing may } \\
\text { occur. Limit is } 2 \\
\text { application/crop }\end{array}$ \\
\hline Botran 5F & 1.6 qts & 3.2 qts & 14 & $\begin{array}{l}\text { Botrytis } \\
\text { Sclerotinia }\end{array}$ & $\begin{array}{l}\text { Some leaf bronzing may occur. } \\
\text { Limit is } 2 \text { applications/crop }\end{array}$ \\
\hline Amistar 80 DF & $5 \mathrm{ozs}$ & 20 ozs & 0 & $\begin{array}{l}\text { Downy mildew } \\
\text { Rhizoctonia } \\
\text { others-see label }\end{array}$ & $\begin{array}{l}\text { Limit is } 1 \text { sequential and } \\
4 \text { applications per crop }\end{array}$ \\
\hline $\begin{array}{l}\text { Champion } \\
\text { Formula } 24.6 \mathrm{~F}\end{array}$ & $1 \frac{1}{3} \mathrm{pts}$ & & & Bacterial disease & \\
\hline
\end{tabular}

Table 9. Insecticides approved for use on insects attacking lettuce.

\begin{tabular}{|c|c|c|c|c|c|c|}
\hline $\begin{array}{l}\text { Trade Name } \\
\text { (Common Name) }\end{array}$ & $\begin{array}{l}\text { Rate } \\
\text { (product/acre) }\end{array}$ & $\begin{array}{l}\text { REI } \\
\text { (hours) }\end{array}$ & $\begin{array}{l}\text { Days to } \\
\text { Harvest }\end{array}$ & Insects & $\begin{array}{l}\text { MOA } \\
\text { Code }^{1}\end{array}$ & Notes \\
\hline $\begin{array}{l}\text { Admire } 2 \mathrm{~F} \\
\text { (imidacloprid) }\end{array}$ & $10-24 \mathrm{fl} \mathrm{oz}$ & 12 & 21 & aphids, whiteflies & $4 \mathrm{~A}$ & $\begin{array}{l}\text { Do not apply more than } 0.38 \mathrm{lb} \text { ai } \\
\text { per acre per year. }\end{array}$ \\
\hline Admire Pro & $4.4-10.5 \mathrm{fl} \mathrm{oz}$ & & & & & \\
\hline $\begin{array}{l}\text { Agree WG } \\
\text { (Bacillus thuringiensis } \\
\text { subspecies aizawai) }\end{array}$ & $0.5-2.0 \mathrm{lb}$ & 4 & 0 & $\begin{array}{l}\text { lepidopteran larvae (cater- } \\
\text { pillar pests) }\end{array}$ & $11 \mathrm{~B} 1$ & $\begin{array}{l}\text { Apply when larvae are small for } \\
\text { best control. Can be used in } \\
\text { greenhouse. OMRI-listed }{ }^{2} \text {. }\end{array}$ \\
\hline $\begin{array}{l}\text { *Agri-Mek } \mathbf{0 . 1 5} \text { EC } \\
\text { (abamectin) }\end{array}$ & $8-16 \mathrm{fl} \mathrm{oz}$ & 12 & 7 & Liriomyza leafminers & 6 & $\begin{array}{l}\text { No more than } 2 \text { sequential appli- } \\
\text { cations. }\end{array}$ \\
\hline $\begin{array}{l}\text { *Ambush 25W } \\
\text { (permethrin) }\end{array}$ & $6.4-12.8 \mathrm{oz}$ & 12 & 1 & $\begin{array}{l}\text { beet armyworm, cabbage } \\
\text { looper, corn earworm, } \\
\text { cutworms, fall armyworm, } \\
\text { leafhoppers, leafminers }\end{array}$ & 3 & $\begin{array}{l}\text { Do not apply more than } 2.0 \mathrm{lb} \\
\text { ai/acre per season. } \\
(128 \mathrm{oz})\end{array}$ \\
\hline $\begin{array}{l}\text { *Ammo } 2.5 \text { EC (cyper- } \\
\text { methrin) }\end{array}$ & $2.5-5 \mathrm{fl} \mathrm{oz}$ & 12 & 5 & $\begin{array}{l}\text { armyworms, corn ear- } \\
\text { worm, crickets, cucumber } \\
\text { beetles, cutworms, flea } \\
\text { beetles, leafhoppers, } \\
\text { loopers, Lygus bug, salt- } \\
\text { marsh caterpillar, stink } \\
\text { bugs, thrips }\end{array}$ & 3 & $\begin{array}{l}\text { Head lettuce only. Maximum of } \\
30 \text { oz/acre per } \\
\text { season. }\end{array}$ \\
\hline $\begin{array}{l}\text { *Asana XL (0.66 EC) } \\
\text { (esfenvalerate) }\end{array}$ & $4.8-9.6 \mathrm{fl} \mathrm{oz}$ & 12 & 7 & $\begin{array}{l}\text { beet armyworm (aids in } \\
\text { control), cabbage looper, } \\
\text { Heliothis spp. }\end{array}$ & 3 & $\begin{array}{l}\text { Head lettuce only. Do not apply } \\
\text { more than } 0.35 \mathrm{lb} \text { a.i. per acre } \\
\text { per season. }\end{array}$ \\
\hline $\begin{array}{l}\text { Assail 70WP } \\
\text { (acetamprid) }\end{array}$ & $0.8-1.7 \mathrm{oz}$ & 12 & 7 & aphids, whiteflies & $4 \mathrm{~A}$ & $\begin{array}{l}\text { Begin applications for whiteflies } \\
\text { when first adults are noticed. Do } \\
\text { not apply more than } 5 \text { times per } \\
\text { season or apply more often than } \\
\text { every } 7 \text { days. }\end{array}$ \\
\hline Assail $30 \mathrm{SG}$ & $2.0-4.0 \mathrm{oz}$ & & & & & \\
\hline $\begin{array}{l}\text { Avaunt } \\
\text { (indoxacarb) }\end{array}$ & $2.5-6.00 z$ & 12 & 3 & $\begin{array}{l}\text { beet armyworm, cabbage } \\
\text { looper, corn earworm }\end{array}$ & 22 & $\begin{array}{l}\text { Do not apply more than } 24 \text { ounc- } \\
\text { es of product per acre per crop. }\end{array}$ \\
\hline
\end{tabular}


Table 9. Continued.

\begin{tabular}{|c|c|c|c|c|c|c|}
\hline $\begin{array}{l}\text { Trade Name } \\
\text { (Common Name) }\end{array}$ & $\begin{array}{l}\text { Rate } \\
\text { (product/acre) }\end{array}$ & $\begin{array}{l}\text { REI } \\
\text { (hours) }\end{array}$ & $\begin{array}{l}\text { Days to } \\
\text { Harvest }\end{array}$ & Insects & $\begin{array}{l}\text { MOA } \\
\text { Code } 1\end{array}$ & Notes \\
\hline $\begin{array}{l}\text { Aza-Direct } \\
\text { (azadirachtin) }\end{array}$ & $\begin{array}{l}1-2 \text { pts, up to } \\
3.5 \text {, if needed }\end{array}$ & 4 & 0 & $\begin{array}{l}\text { aphids, beetles, caterpil- } \\
\text { lars, leafhoppers, leafmin- } \\
\text { ers, mites, stink bugs, } \\
\text { thrips, weevils, whiteflies }\end{array}$ & 26 & $\begin{array}{l}\text { Antifeedant, repellant, insect } \\
\text { growth regulator. OMRI-listed. }\end{array}$ \\
\hline $\begin{array}{l}\text { Azatin XL } \\
\text { (azadirachtin) }\end{array}$ & $5-21 \mathrm{fl} \mathrm{oz}$ & 4 & 0 & $\begin{array}{l}\text { aphids, beetles, caterpil- } \\
\text { lars, leafhoppers, leafmin- } \\
\text { ers, thrips, weevils, } \\
\text { whiteflies }\end{array}$ & 26 & $\begin{array}{l}\text { Antifeedant, repellant, insect } \\
\text { growth regulator. }\end{array}$ \\
\hline $\begin{array}{l}\text { *Baythroid } 2 \\
\text { (cyfluthrin) }\end{array}$ & $0.8-3.2 \mathrm{fl} \mathrm{oz}$ & 12 & 0 & $\begin{array}{l}\text { beet armyworm (1st and } \\
2^{\text {nd }} \text { instars), cabbage } \\
\text { looper, corn earworm, } \\
\text { cutworms, flea beetles, } \\
\text { grasshoppers, potato } \\
\text { leafhopper, saltmarsh cat- } \\
\text { erpillar, thrips, vegetable } \\
\text { weevil, yellowstriped } \\
\text { armyworm }\end{array}$ & 3 & $\begin{array}{l}\text { Apply no more than } 4 \text { times per } \\
\text { season. }\end{array}$ \\
\hline $\begin{array}{l}\text { Biobit HP } \\
\text { (Bacillus thuringiensis } \\
\text { subspecies kurstaki) }\end{array}$ & $0.5-2.0 \mathrm{lb}$ & 4 & 0 & $\begin{array}{l}\text { caterpillars (will not con- } \\
\text { trol large armyworms) }\end{array}$ & 11B2 & $\begin{array}{l}\text { Treat when larvae are young. } \\
\text { Good coverage is essential. } \\
\text { Can be used in the greenhouse. } \\
\text { OMRI-listed. }\end{array}$ \\
\hline $\begin{array}{l}\text { BotaniGard } 22 \text { WP, ES } \\
\text { (Beauveria bassiana) }\end{array}$ & $\begin{array}{l}\text { WP: } \\
0.5-2 \text { Ib/100 } \\
\text { gal } \\
\text { ES: } \\
0.5-2 \text { qts/100 } \\
\text { gal }\end{array}$ & 4 & 0 & aphids, thrips, whiteflies & -- & $\begin{array}{l}\text { May be used in greenhouses. } \\
\text { Contact dealer for recommen- } \\
\text { dations if an adjuvant must be } \\
\text { used. Not compatible in tank mix } \\
\text { with fungicides. }\end{array}$ \\
\hline $\begin{array}{l}\text { *Capture 2EC } \\
\text { (bifenthrin) }\end{array}$ & $2.1-6.4 \mathrm{fl} \mathrm{oz}$ & 12 & 7 & $\begin{array}{l}\text { aphids, armyworms, } \\
\text { carmine mite, corn ear- } \\
\text { worm, cucumber beetle, } \\
\text { cutworms, flea beetles, } \\
\text { leafhoppers, Lygus spp., } \\
\text { saltmarsh caterpillar, stink } \\
\text { bug spp., twospotted spi- } \\
\text { der mite, whiteflies }\end{array}$ & 3 & Head lettuce only. \\
\hline $\begin{array}{l}\text { Confirm 2F } \\
\text { (tebufenozide) }\end{array}$ & 6-8 fl oz & 4 & 7 & $\begin{array}{l}\text { armyworms, cabbage } \\
\text { looper, garden webworm }\end{array}$ & 18 & $\begin{array}{l}\text { Do not exceed } 56 \text { ounces of } \\
\text { product per acre per season. }\end{array}$ \\
\hline $\begin{array}{l}\text { Courier 70WP, 40SC } \\
\text { (buprofezin) }\end{array}$ & $\begin{array}{l}\text { 70WP: } \\
\text { 6-9 oz } \\
\text { 40SC: } \\
9-13.6 \mathrm{fl} \mathrm{oz}\end{array}$ & 12 & 7 & whitefly nymphs & 16 & $\begin{array}{l}\text { Insect growth regulator. Do not } \\
\text { make more than } 2 \text { applications } \\
\text { per season per crop. Allow } 7 \\
\text { days between applications. Do } \\
\text { not plant food crops except those } \\
\text { on the label within } 120 \text { days fol- } \\
\text { lowing application. }\end{array}$ \\
\hline $\begin{array}{l}\text { Crymax WDG } \\
\text { (Bacillus thuringiensis } \\
\text { subspecies kurstaki) }\end{array}$ & $0.5-2.0 \mathrm{lb}$ & 4 & 0 & caterpillars & $11 \mathrm{~B} 2$ & $\begin{array}{l}\text { Use high rate for armyworms. } \\
\text { Treat when larvae are young. }\end{array}$ \\
\hline $\begin{array}{l}\text { Deliver } \\
\text { (Bacillus thuringiensis } \\
\text { subspecies kurstaki) }\end{array}$ & $0.25-1.5 \mathrm{lb}$ & 4 & 0 & caterpillars & 11B2 & $\begin{array}{l}\text { Use higher rates for armyworms. } \\
\text { OMRI-listed?2. }\end{array}$ \\
\hline
\end{tabular}


Table 9. Continued.

\begin{tabular}{|c|c|c|c|c|c|c|}
\hline $\begin{array}{l}\text { Trade Name } \\
\text { (Common Name) }\end{array}$ & $\begin{array}{l}\text { Rate } \\
\text { (product/acre) }\end{array}$ & $\begin{array}{l}\text { REI } \\
\text { (hours) }\end{array}$ & $\begin{array}{l}\text { Days to } \\
\text { Harvest }\end{array}$ & Insects & $\begin{array}{l}\text { MOA } \\
\text { Code }^{1}\end{array}$ & Notes \\
\hline \multirow[t]{2}{*}{$\begin{array}{l}\text { *Diazinon } 4 \mathrm{E}, \text { *50W } \\
\text { (diazinon) }\end{array}$} & $\begin{array}{l}\text { foliar: } \\
\text { AG500, 4E: } \\
0.5-1 \mathrm{pt} \\
\text { 50W: } \\
0.5-1 \mathrm{lb}\end{array}$ & 24 & $\begin{array}{l}14 \\
\text { (foliar) }\end{array}$ & aphids, leafminers & $1 \mathrm{~B}$ & Do not apply more than 5 times. \\
\hline & $\begin{array}{l}\text { preplant: } \\
\text { AG500, 4E: } \\
\text { 1-4 qts } \\
\text { 50W: 2-8 lb }\end{array}$ & 24 & preplant & $\begin{array}{l}\text { cutworms, mole crickets, } \\
\text { wireworms }\end{array}$ & $1 \mathrm{~B}$ & See label. \\
\hline $\begin{array}{l}\text { Dimethoate } 4 \text { EC, } 2.67 \\
\text { (dimethoate) }\end{array}$ & $\begin{array}{l}\text { 4EC: } \\
0.5 \mathrm{pt} \\
2.67: \\
0.75\end{array}$ & 48 & $14=$ leaf & $\begin{array}{l}\text { f aphids, leafhoppers, } \\
\text { leafminers }\end{array}$ & $1 \mathrm{~B}$ & $\begin{array}{l}\text { Leaf lettuce only, not for head } \\
\text { lettuce. }\end{array}$ \\
\hline $\begin{array}{l}\text { DiPel DF } \\
\text { (Bacillus thuringiensis } \\
\text { subspecies kurstaki) }\end{array}$ & $0.5-2.0 \mathrm{lb}$ & 4 & 0 & caterpillars & 11B2 & $\begin{array}{l}\text { Treat when larvae are young. } \\
\text { Good coverage is essential. Can } \\
\text { be used in greenhouse. OMRI- } \\
\text { listed. }\end{array}$ \\
\hline $\begin{array}{l}\text { *Di-Syston } 8 \text { EC (disul- } \\
\text { foton) }\end{array}$ & - $1-2 \mathrm{pt}$ & 48 & 60 & $\begin{array}{l}\text { aphids, leafhoppers, } \\
\text { mites, root aphids, thrips }\end{array}$ & $1 \mathrm{~B}$ & $\begin{array}{l}\text { Do not apply to transplanted let- } \\
\text { tuce. }\end{array}$ \\
\hline $\begin{array}{l}\text { Endosulfan 3EC } \\
\text { (endosulfan) }\end{array}$ & $1-1.33$ qts & 24 & 14 & $\begin{array}{l}\text { aphids, armyworms, cab- } \\
\text { bage looper, leafhoppers, } \\
\text { whiteflies }\end{array}$ & 2 & $\begin{array}{l}\text { Head lettuce - no more than } 3 \\
\text { applications after thinning. } \\
\text { Leaf lettuce - no more than two } \\
\text { applications per year. }\end{array}$ \\
\hline $\begin{array}{l}\text { Entrust } \\
\text { (spinosad) }\end{array}$ & $0.5-3 \mathrm{oz}$ & 4 & 1 & $\begin{array}{l}\text { armyworms, cabbage } \\
\text { looper, leafminers, thrips }\end{array}$ & 5 & $\begin{array}{l}\text { See label for resistance manage- } \\
\text { ment recommendations. Do not } \\
\text { apply more than } 9 \text { oz per acre } \\
\text { per crop, or more than } 3 \text { times in } \\
21 \text { days. OMRI-listed². }\end{array}$ \\
\hline $\begin{array}{l}\text { Extinguish } \\
\text { ((S)-methoprene) }\end{array}$ & $1-1.5 \mathrm{lb}$ & 0 & 0 & fire ants & $7 \mathrm{~A}$ & $\begin{array}{l}\text { Slow-acting IGR (insect growth } \\
\text { regulator). Best applied early } \\
\text { spring and fall where crop will be } \\
\text { grown. Colonies will be reduced } \\
\text { after three weeks and eliminated } \\
\text { after } 8 \text { to } 10 \text { weeks. May be } \\
\text { applied by ground equipment or } \\
\text { aerially. }\end{array}$ \\
\hline $\begin{array}{l}\text { Fulfill } \\
\text { (pymetrozine) }\end{array}$ & $2.75 \mathrm{oz}$ & 12 & 7 & $\begin{array}{l}\text { aphids, suppression of } \\
\text { whiteflies }\end{array}$ & $9 \mathrm{~B}$ & $\begin{array}{l}\text { Do not exceed } 5.5 \text { oz product per } \\
\text { acre per season. }\end{array}$ \\
\hline $\begin{array}{l}\text { Intrepid 2F } \\
\text { (methoxyfenozide) }\end{array}$ & $4-16 \mathrm{fl} \mathrm{oz}$ & 4 & 1 & $\begin{array}{l}\text { armyworms, cabbage } \\
\text { looper, webworms }\end{array}$ & 18 & $\begin{array}{l}\text { Do not apply more than } 64 \mathrm{fl} \mathrm{oz/} \\
\text { acre per season. }\end{array}$ \\
\hline $\begin{array}{l}\text { Javelin WG } \\
\text { (Bacillus thuringiensis } \\
\text { subspecies kurstaki) }\end{array}$ & $0.12-1.5 \mathrm{lb}$ & 4 & 0 & $\begin{array}{l}\text { most caterpillars, but } \\
\text { not Spodoptera species } \\
\text { (armyworms) }\end{array}$ & 11B2 & $\begin{array}{l}\text { Treat when larvae are young. } \\
\text { Thorough coverage is essential. } \\
\text { OMRI-listed?2. }\end{array}$ \\
\hline $\begin{array}{l}\text { Kryocide } \\
\text { (cryolite) }\end{array}$ & $8-20 \mathrm{lb}$ & 12 & 14 & $\begin{array}{l}\text { armyworms, cabbage } \\
\text { looper, corn earworm, } \\
\text { tobacco budworm }\end{array}$ & $9 \mathrm{~A}$ & $\begin{array}{l}\text { Do not apply more than } 160 \text { 1b/ } \\
\text { acre/season. }\end{array}$ \\
\hline $\begin{array}{l}\text { *Lannate LV; *SP } \\
\text { (methomyl) }\end{array}$ & $\begin{array}{l}\text { LV: } 0.75-3 \mathrm{pt} \\
\text { SP: } 0.25-1.0 \mathrm{lb}\end{array}$ & 48 & $\begin{array}{l}7 \text { or } 10 \text {, } \\
\text { depend- } \\
\text { ing on } \\
\text { rate } \\
\text { used }\end{array}$ & $\begin{array}{l}\text { aphids, aster leafhopper, } \\
\text { beet armyworm, cabbage } \\
\text { looper, corn earworm, } \\
\text { thrips, variegated cut- } \\
\text { worm }\end{array}$ & $1 \mathrm{~A}$ & \\
\hline
\end{tabular}


Table 9. Continued.

\begin{tabular}{|c|c|c|c|c|c|c|}
\hline $\begin{array}{l}\text { Trade Name } \\
\text { (Common Name) }\end{array}$ & $\begin{array}{l}\text { Rate } \\
\text { (product/acre) }\end{array}$ & $\begin{array}{l}\text { REI } \\
\text { (hours) }\end{array}$ & $\begin{array}{l}\text { Days to } \\
\text { Harvest }\end{array}$ & Insects & $\begin{array}{l}\text { MOA } \\
\text { Code }^{1}\end{array}$ & Notes \\
\hline $\begin{array}{l}\text { *Larvin } 3.2 \\
\text { (thiodicarb) }\end{array}$ & $16-30 \mathrm{fl} \mathrm{oz}$ & 48 & 14 & $\begin{array}{l}\text { armyworms, beet army- } \\
\text { worm, cabbage looper, } \\
\text { corn earworm, fall army- } \\
\text { worm, tomato fruitworm, } \\
\text { southern armyworm }\end{array}$ & $1 \mathrm{~A}$ & $\begin{array}{l}\text { Do not exceed } 1.5 \mathrm{lb} \text { active ingre- } \\
\text { dient per acre per season }(60 \mathrm{fl} \\
\text { 0z). }\end{array}$ \\
\hline $\begin{array}{l}\text { Lepinox WDG } \\
\text { (Bacillus thuringiensis } \\
\text { subspecies kurstaki) }\end{array}$ & $1.0-2.0 \mathrm{lb}$ & 12 & 0 & $\begin{array}{l}\text { for most caterpillars, } \\
\text { including beet armyworm } \\
\text { (see label) }\end{array}$ & 11B2 & $\begin{array}{l}\text { Treat when larvae are small. } \\
\text { Thorough coverage is essential. }\end{array}$ \\
\hline $\begin{array}{l}\text { Malathion 8F } \\
\text { (malathion) }\end{array}$ & $2 \mathrm{pt}$ & 12 & $\begin{array}{l}\text { head - } 7 \\
\text { leaf - } \\
14\end{array}$ & $\begin{array}{l}\text { aphids, cabbage looper, } \\
\text { leafhoppers, mites }\end{array}$ & $1 \mathrm{~B}$ & $\begin{array}{l}\text { Can be used in } \\
\text { greenhouse. }\end{array}$ \\
\hline $\begin{array}{l}\text { *MSR Spray } \\
\text { Concentrate (oxy- } \\
\text { demeton-methyl) }\end{array}$ & $1.5-2 \mathrm{pt}$ & 48 & 21 & aphids, mites & $1 \mathrm{~B}$ & For head lettuce only. \\
\hline $\begin{array}{l}\text { M-Pede } \mathbf{4 9} \% \\
\text { Soap, insecticidal }\end{array}$ & $1-2 \%$ V/V & 12 & 0 & $\begin{array}{l}\text { aphids, leafhoppers, } \\
\text { mites, plant bugs, thrips, } \\
\text { whiteflies }\end{array}$ & -- & OMRI-listed². \\
\hline $\begin{array}{l}\text { *Mustang Max } \\
\text { (zeta-cypermethrin) }\end{array}$ & $2.24-4.0 \mathrm{oz}$ & 12 & 5 & $\begin{array}{l}\text { armyworms, corn ear- } \\
\text { worm, crickets, cucumber } \\
\text { beetles, cutworms, flea } \\
\text { beetles, leafhoppers, } \\
\text { loopers, saltmarsh cater- } \\
\text { pillar, stink bugs }\end{array}$ & 3 & Head lettuce only. \\
\hline $\begin{array}{l}\text { Neemix } 4.5 \text { EC (azadi- } \\
\text { rachtin) }\end{array}$ & $4-16 \mathrm{fl} \mathrm{oz}$ & 12 & 0 & $\begin{array}{l}\text { aphids, armyworms, cab- } \\
\text { bage looper, cutworms, } \\
\text { leafminers, thrips, white- } \\
\text { flies }\end{array}$ & 26 & OMRI-listed². \\
\hline $\begin{array}{l}\text { Oberon 2SC } \\
\text { (spiromesifen) }\end{array}$ & $7.0-8.5 \mathrm{fl} \mathrm{oz}$ & 12 & 7 & whiteflies & 23 & $\begin{array}{l}\text { Maximum amount per crop: } 25.5 \\
\text { fl oz. No more than } 3 \text { applica- } \\
\text { tions. }\end{array}$ \\
\hline $\begin{array}{l}\text { Orthene } 75 \mathrm{~S} \\
\text { (acephate) }\end{array}$ & $0.67-1.33 \mathrm{lb}$ & 24 & 21 & $\begin{array}{l}\text { aphids, armyworms, } \\
\text { aster leafhopper, cabbage } \\
\text { looper }\end{array}$ & $1 \mathrm{~B}$ & $\begin{array}{l}\text { Winter crops in Florida. Head let- } \\
\text { tuce only. }\end{array}$ \\
\hline $\begin{array}{l}\text { *Pounce } 3.2 \text { EC (per- } \\
\text { methrin) }\end{array}$ & $2-80 z$ & 12 & 1 & $\begin{array}{l}\text { aphids, armyworms, } \\
\text { beet armyworm, cabbage } \\
\text { looper, corn earworm, } \\
\text { cutworms, fall armyworm, } \\
\text { leafhoppers, leafminers, } \\
\text { southern armyworm, } \\
\text { tobacco budworm }\end{array}$ & 3 & \\
\hline $\begin{array}{l}\text { *Proaxis Insecticide } \\
\text { (gamma-cyhalothrin) }\end{array}$ & $1.92-3.84 \mathrm{fl} \mathrm{oz}$ & 24 & 1 & $\begin{array}{l}\text { Aphids(1), armyworm, } \\
\text { beet armyworm(2), } \\
\text { cabbage looper, corn } \\
\text { earworm, cutworm, fall } \\
\text { armyworm(2), flea beetles, } \\
\text { grasshoppers, green } \\
\text { cloverworm, leafhop- } \\
\text { pers, meadow spittlebug, } \\
\text { southern armyworm, } \\
\text { spider mites (1), stink } \\
\text { bugs, tobacco budworm, } \\
\text { vegetable weevil (adult), } \\
\text { whiteflies(1) }\end{array}$ & 3 & $\begin{array}{l}\text { (1) Suppression only. } \\
\text { (2) First and second instars only. } \\
\text { Do not apply more than } 2.4 \text { pints } \\
\text { per acre per season. }\end{array}$ \\
\hline $\begin{array}{l}\text { *Proclaim } \\
\text { (emamectin benzoate) }\end{array}$ & $2.4-4.8 \mathrm{oz}$ & 48 & 7 & $\begin{array}{l}\text { beet armyworm, corn } \\
\text { earworm, fall armyworm, } \\
\text { Liriomyza leafminers } \\
\text { (suppression), loopers, } \\
\text { tobacco budworm }\end{array}$ & 6 & $\begin{array}{l}\text { Do not make more than } 2 \\
\text { sequential applications without } \\
\text { rotation to another product with a } \\
\text { different mode of action. }\end{array}$ \\
\hline
\end{tabular}


Table 9. Continued.

\begin{tabular}{|c|c|c|c|c|c|c|}
\hline $\begin{array}{l}\text { Trade Name } \\
\text { (Common Name) }\end{array}$ & $\begin{array}{l}\text { Rate } \\
\text { (product/acre) }\end{array}$ & $\begin{array}{l}\text { REI } \\
\text { (hours) }\end{array}$ & $\begin{array}{l}\text { Days to } \\
\text { Harvest }\end{array}$ & Insects & $\begin{array}{l}\text { MOA } \\
\text { Code }^{1}\end{array}$ & Notes \\
\hline $\begin{array}{l}\text { Provado } 1.6 \mathrm{~F} \text { (imida- } \\
\text { cloprid) }\end{array}$ & $3.80 z$ & 12 & 7 & $\begin{array}{l}\text { aphids, flea beetles, } \\
\text { whiteflies }\end{array}$ & $4 \mathrm{~A}$ & \\
\hline $\begin{array}{l}\text { Pyrellin EC } \\
\text { (pyrethrin + rotenone) }\end{array}$ & $1-2 \mathrm{pt}$ & 12 & $\begin{array}{l}12 \\
\text { hours }\end{array}$ & $\begin{array}{l}\text { aphids, beet webworm, } \\
\text { cabbage looper, cucumber } \\
\text { beetle, flea beetles, leaf- } \\
\text { hoppers, leafminers, loop- } \\
\text { ers, lygus bugs, mites, } \\
\text { plant bugs, stink bugs, } \\
\text { thrips, vegetable weevil, } \\
\text { whiteflies }\end{array}$ & 3,21 & \\
\hline $\begin{array}{l}\text { Sevin 80S, XLR, 4F } \\
\text { (carbaryl) }\end{array}$ & $\begin{array}{l}\text { 80S: } 0.63-2.5 \\
\text { Ib } \\
\text { XLR, } 4 F: 0.5- \\
2.0 \text { qt }\end{array}$ & 12 & 14 & $\begin{array}{l}\text { armyworms, aster leaf- } \\
\text { hopper, corn earworm, } \\
\text { fall armyworm, flea bee- } \\
\text { tles, leafhoppers, lygus } \\
\text { bugs, spittlebugs, stink } \\
\text { bugs, tarnished plant bug }\end{array}$ & $1 \mathrm{~A}$ & $\begin{array}{l}\text { Repeat as needed, up to } 5 \text { times, } \\
\text { at least } 7 \text { days apart. }\end{array}$ \\
\hline $\begin{array}{l}\text { SpinTor } 2 \text { SC } \\
\text { (spinosad) }\end{array}$ & $1.5-10 \mathrm{fl} \mathrm{oz}$ & 4 & 1 & $\begin{array}{l}\text { armyworms, cabbage } \\
\text { looper, leafminers } \\
\text { (Liriomyza spp.), thrips }\end{array}$ & 5 & $\begin{array}{l}\text { Do not apply more than } 29 \mathrm{fl} \mathrm{oz} \\
\text { per acre per crop. }\end{array}$ \\
\hline $\begin{array}{l}\text { Sun Spray } \mathbf{9 8 . 8 \%} \text {, } \\
\text { JMS Stylet-0il, others } \\
\text { Oil, insecticidal }\end{array}$ & 3-6 qts (JMS) & 4 & 0 & $\begin{array}{l}\text { leafhoppers, leafminers, } \\
\text { mites, thrips, whiteflies }\end{array}$ & -- & $\begin{array}{l}\text { Organic Stylet-Oil is } \\
\text { OMRI-listed }{ }^{2} \text {. }\end{array}$ \\
\hline $\begin{array}{l}\text { *Telone C-35 (dichloro- } \\
\text { propene + chloropicrin) }\end{array}$ & See label & $\begin{array}{l}5 \text { days } \\
\text { - See } \\
\text { label }\end{array}$ & preplant & symphylans, wireworms & -- & $\begin{array}{l}\text { See supplemental label for use } \\
\text { restrictions in south and central } \\
\text { Florida. }\end{array}$ \\
\hline \multicolumn{7}{|l|}{$\begin{array}{l}{ }^{*} \text { Telone II } \\
\text { (dichloropropene) }\end{array}$} \\
\hline $\begin{array}{l}\text { Trigard } \\
\text { (cryomazine) }\end{array}$ & $2.66 \mathrm{oz}$ & 12 & 7 days & leafminers & 17 & $\begin{array}{l}\text { Limited to six applications for } \\
\text { head lettuce and five applications } \\
\text { for leaf lettuce. }\end{array}$ \\
\hline $\begin{array}{l}\text { Trilogy } \\
\text { (extract of neem oil) }\end{array}$ & $0.5-2.0 \% \mathrm{~V} / \mathrm{V}$ & 4 & 0 & $\begin{array}{l}\text { aphids, mites, suppres- } \\
\text { sion of thrips and white- } \\
\text { flies }\end{array}$ & 26 & $\begin{array}{l}\text { Apply morning or evening to } \\
\text { reduce potential for leaf burn. } \\
\text { Toxic to bees exposed to direct } \\
\text { treatment. } \\
\text { OMRI-listed. }\end{array}$ \\
\hline $\begin{array}{l}\text { Venom } \\
\text { (dinotefuran) }\end{array}$ & $\begin{array}{l}\text { foliar: } 1-3 \text { oz } \\
\text { soil: } 5-6 \text { oz }\end{array}$ & 12 & $\begin{array}{l}\text { foliar } \\
-7 \\
\text { soil - } 21\end{array}$ & $\begin{array}{l}\text { green peach aphid, } \\
\text { leafhoppers, leafminers, } \\
\text { potato aphid, whiteflies }\end{array}$ & $4 \mathrm{~A}$ & $\begin{array}{l}\text { Do not apply more than } 6 \text { oz per } \\
\text { acre per season (foliar) or } 12 \mathrm{oz} \\
\text { per acre per season (soil). Do not } \\
\text { use both application methods. }\end{array}$ \\
\hline $\begin{array}{l}\text { *Warrior (lambda-cyh- } \\
\text { alothrin) }\end{array}$ & $1.92-3.84 \mathrm{fl} \mathrm{oz}$ & 24 & 1 & $\begin{array}{l}\text { aphids(1), armyworms, } \\
\text { beet armyworm, cabbage } \\
\text { looper, corn earworm, } \\
\text { cutworms, fall armyworm, } \\
\text { flea beetles, grasshop- } \\
\text { pers, leafhoppers, mites, } \\
\text { plant bugs, saltmarsh } \\
\text { caterpillar, southern army- } \\
\text { worm, spittlebugs, stink } \\
\text { bugs, tobacco budworm, } \\
\text { vegetable weevil }\end{array}$ & 3 & $\begin{array}{l}\text { Head and leaf. Do not apply more } \\
\text { than } 0.3 \mathrm{lb} \text { ai/acre per } \\
\text { season. } \\
\text { (1) Suppression only. }\end{array}$ \\
\hline $\begin{array}{l}\text { Xentari DF } \\
\text { (Bacillus thuringiensis } \\
\text { subspecies aizawai) }\end{array}$ & $0.5-2.0 \mathrm{lb}$ & 4 & 0 & caterpillars & $11 \mathrm{~B} 1$ & $\begin{array}{l}\text { Treat when larvae are young. } \\
\text { Thorough coverage is essential. } \\
\text { May be used in the greenhouse. } \\
\text { Can be used in organic produc- } \\
\text { tion. }\end{array}$ \\
\hline
\end{tabular}


Table 9. Continued.

\begin{tabular}{|c|c|c|c|}
\hline $\begin{array}{l}\text { Trade Name } \\
\text { (Common Name) }\end{array}$ & $\begin{array}{ll}\text { Rate } & \text { REI } \\
\text { (product/acre) } & \text { (hours) } \\
\end{array}$ & $\begin{array}{l}\text { Days to } \\
\text { Harvest Insects }\end{array}$ & Notes \\
\hline \multicolumn{4}{|c|}{$\begin{array}{l}\text { The pesticide information presented in this table was current with federal and state regulations at the time of revision. The user is respon } \\
\text { sible for determining the intended use is consistent with the label of the product being used. Use pesticides safely. Read and follow label } \\
\text { instructions. }\end{array}$} \\
\hline \multirow{2}{*}{\multicolumn{4}{|c|}{$\begin{array}{l}{ }^{1} \text { Mode of Action codes for vegetable pest insecticides from the Insecticide Resistance Action Committee (IRAC) Mode of Action } \\
\text { Classification v.3.3 October 2003. 1A. Acetylcholine esterase inhibitors, Carbamates 1B. Acetylcholine esterase inhibi- } \\
\text { tors, Organophosphates }\end{array}$}} \\
\hline & & & \\
\hline 2A. GABA-gat & \multicolumn{3}{|c|}{ GABA-gated chloride channel antagonists } \\
\hline & \multicolumn{3}{|c|}{ Sodium channel modulators } \\
\hline Nicotinic & \multirow{2}{*}{\multicolumn{3}{|c|}{$\begin{array}{l}\text { Nicotinic Acetylcholine receptor agonists/antagonists, Neonicotinoids } \\
\text { Nicotinic Acetylcholine receptor agonists (not group 4) }\end{array}$}} \\
\hline Nicotinic & & & \\
\hline Chloride & \multicolumn{3}{|c|}{ Chloride channel activators } \\
\hline Juvenile $\mathrm{r}$ & \multicolumn{3}{|c|}{ Juvenile hormone mimics, Juvenile hormone analogues } \\
\hline 7D. Juvenile I & \multicolumn{3}{|c|}{ Juvenile hormone mimics, Pyriproxifen } \\
\hline \multirow{2}{*}{\multicolumn{4}{|c|}{ Compounds of unknown or non-specific mode of action (selective feeding blockers), Cryolite }} \\
\hline \multicolumn{3}{|c|}{ Compounds of unknown or non-specific mode of action (selective feeding blockers), Pymetrozine } & \\
\hline 11B1. Microbial & \multicolumn{3}{|c|}{ Microbial disruptors of insect midgut membranes, B.t. var aizawai } \\
\hline 11B2. Microbial & \multicolumn{3}{|c|}{ Microbial disruptors of insect midgut membranes, B.t. var kurstaki } \\
\hline \multirow{2}{*}{\multicolumn{4}{|c|}{ Inhibitors of oxidative phosphorylation, disruptors of ATP formation, Organotin miticide }} \\
\hline 15. Inhibitors & & & \\
\hline \multicolumn{4}{|c|}{ Inhibitors of chitin biosynthesis, type 1, Homopteran } \\
\hline Inhibitors & \multicolumn{3}{|c|}{ Inhibitors of chitin biosynthesis, type 2, Dipteran } \\
\hline Ecdysone & \multicolumn{3}{|c|}{ Ecdysone agonist/disruptor } \\
\hline Site II ele & \multicolumn{3}{|c|}{ Site II electron transport inhibitors } \\
\hline Site I ele & \multicolumn{3}{|c|}{ Site I electron transport inhibitors } \\
\hline Voltage- $c$ & \multicolumn{3}{|c|}{ Voltage-dependent sodium channel blocker } \\
\hline Inhibitors & \multicolumn{3}{|c|}{ Inhibitors of lipid biosynthesis } \\
\hline & \multicolumn{3}{|c|}{ Neuroactive (unknown mode of action) } \\
\hline Unknown & \multicolumn{3}{|c|}{ Unknown mode of action, Azadirachtin } \\
\hline \multicolumn{4}{|c|}{2 OMRI listed: Listed by the Organic Materials Review Institute for use in organic production. } \\
\hline${ }^{*}$ Restricted Use 0 & & & \\
\hline
\end{tabular}


Table 10. Selected insecticides approved for use on insects attacking endive and escarole.

\begin{tabular}{|c|c|c|c|c|c|c|}
\hline $\begin{array}{l}\text { Trade Name } \\
\text { (Common Name) }\end{array}$ & $\begin{array}{l}\text { Rate } \\
\text { (product/acre) }\end{array}$ & $\begin{array}{l}\text { REI } \\
\text { (hours) }\end{array}$ & $\begin{array}{l}\text { Days to } \\
\text { Harvest }\end{array}$ & Insects & $\begin{array}{l}\text { MOA } \\
\text { Code }^{1}\end{array}$ & Notes \\
\hline $\begin{array}{l}\text { Admire } 2 \mathrm{~F} \\
\text { (imidacloprid) }\end{array}$ & $10-24 \mathrm{fl} \mathrm{oz}$ & 12 & 21 & aphids, whiteflies & $4 \mathrm{~A}$ & $\begin{array}{l}\text { Do not apply more than } 0.38 \mathrm{lb} \text { ai } \\
\text { per acre per year. }\end{array}$ \\
\hline Admire Pro & $4.4-10.5 \mathrm{fl} \mathrm{oz}$ & & & & & \\
\hline $\begin{array}{l}\text { Agree WG } \\
\text { (Bacillus thuringiensis } \\
\text { subspecies aizawai) }\end{array}$ & $0.5-2.0 \mathrm{lb}$ & 4 & 0 & $\begin{array}{l}\text { lepidopteran larvae (cater- } \\
\text { pillar pests) }\end{array}$ & $11 \mathrm{~B} 1$ & $\begin{array}{l}\text { Apply when larvae are small for } \\
\text { best control. OMRI-listed². }\end{array}$ \\
\hline $\begin{array}{l}\text { *Agri-Mek 0.15EC } \\
\text { (abamectin) }\end{array}$ & $8-16 \mathrm{fl} \mathrm{oz}$ & 12 & 7 & $\begin{array}{l}\text { Liriomyza leafminers, spi- } \\
\text { der mites }\end{array}$ & 6 & $\begin{array}{l}\text { No more than } 2 \text { sequential appli- } \\
\text { cations. Maximum of } 48 \text { oz per } \\
\text { acre per season. }\end{array}$ \\
\hline $\begin{array}{l}\text { *Ambush 25W } \\
\text { (permethrin) }\end{array}$ & $6.4-12.8 \mathrm{oz}$ & 12 & 1 & $\begin{array}{l}\text { beet armyworm, cabbage } \\
\text { looper, corn earworm, } \\
\text { cutworms, fall armyworm, } \\
\text { leafhoppers, leafminers }\end{array}$ & 3 & $\begin{array}{l}\text { Do not apply more than } 2.0 \mathrm{lb} \\
\text { ai/acre per season. } \\
(128 \mathrm{oz})\end{array}$ \\
\hline $\begin{array}{l}\text { Assail 70WP } \\
\text { (acetamiprid) }\end{array}$ & $0.8-1.70 z$ & 12 & 7 & aphids, whiteflies & $4 \mathrm{~A}$ & $\begin{array}{l}\text { Do not apply more than } 5 \text { times } \\
\text { per season or more often than } \\
\text { every } 7 \text { days. }\end{array}$ \\
\hline Assail $30 \mathrm{SG}$ & $2.0-4.0 \mathrm{oz}$ & & & & & \\
\hline $\begin{array}{l}\text { Aza-Direct } \\
\text { (azadirachtin) }\end{array}$ & $\begin{array}{l}1-2 \text { pts, up to } \\
3.5 \text {, if needed }\end{array}$ & 4 & 0 & $\begin{array}{l}\text { aphids, beetles, caterpil- } \\
\text { lars, leafhoppers, leafmin- } \\
\text { ers, mites, stink bugs, } \\
\text { thrips, weevils, whiteflies }\end{array}$ & 26 & $\begin{array}{l}\text { Antifeedant, repellent, insect } \\
\text { growth regulator. OMRI-listed². }\end{array}$ \\
\hline $\begin{array}{l}\text { Azatin XL } \\
\text { (azadirachtin) }\end{array}$ & $5-21 \mathrm{fl} \mathrm{oz}$ & 4 & 0 & $\begin{array}{l}\text { aphids, beetles, caterpil- } \\
\text { lars, leafhoppers, leafmin- } \\
\text { ers, thrips, weevils, } \\
\text { whiteflies }\end{array}$ & 26 & $\begin{array}{l}\text { Antifeedant, repellent, insect } \\
\text { growth regulator. }\end{array}$ \\
\hline $\begin{array}{l}\text { Baythroid } 2 \\
\text { (cyfluthrin) }\end{array}$ & $0.8-3.2 \mathrm{fl} \mathrm{oz}$ & 12 & 0 & 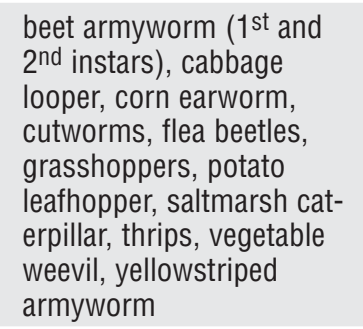 & 3 & $\begin{array}{l}\text { Apply no more than } 4 \text { times per } \\
\text { season. }\end{array}$ \\
\hline $\begin{array}{l}\text { Biobit HP } \\
\text { (Bacillus thuringiensis } \\
\text { subspecies kurstaki) }\end{array}$ & $0.5-2.0 \mathrm{lb}$ & 4 & 0 & $\begin{array}{l}\text { caterpillars (will not con- } \\
\text { trol large armyworms) }\end{array}$ & $11 \mathrm{~B} 2$ & $\begin{array}{l}\text { Treat when larvae are young. } \\
\text { Good coverage is essential. Can } \\
\text { be used in the greenhouse. OMRI- } \\
\text { listed }^{2} \text {. }\end{array}$ \\
\hline $\begin{array}{l}\text { BotaniGard } 22 \text { WP, ES } \\
\text { (Beauveria bassiana) }\end{array}$ & $\begin{array}{l}\text { WP: } \\
0.5-2 \mathrm{lb} / 100 \mathrm{gal} \\
\text { ES: } \\
0.5-2 \mathrm{qts} / 100 \mathrm{ga}\end{array}$ & 4 & 0 & aphids, thrips, whiteflies & -- & $\begin{array}{l}\text { May be used in greenhouses. } \\
\text { Contact dealer for recommenda- } \\
\text { tions if an adjuvant must be used. } \\
\text { Not compatible in tank mix with } \\
\text { fungicides. }\end{array}$ \\
\hline $\begin{array}{l}\text { Confirm 2F } \\
\text { (tebufenozide) }\end{array}$ & $6-8 \mathrm{fl} \mathrm{oz}$ & 4 & 7 & $\begin{array}{l}\text { armyworms, cabbage } \\
\text { looper, garden webworm }\end{array}$ & 18 & $\begin{array}{l}\text { Do not exceed } 56 \text { ounce of prod- } \\
\text { uct per season. }\end{array}$ \\
\hline $\begin{array}{l}\text { Crymax WDG } \\
\text { (Bacillus thuringiensis } \\
\text { subspecies kurstaki) }\end{array}$ & $0.5-2.0 \mathrm{lb}$ & 4 & 0 & caterpillars & $11 \mathrm{~B} 2$ & $\begin{array}{l}\text { Use high rate for armyworms. } \\
\text { Treat when larvae are young. }\end{array}$ \\
\hline
\end{tabular}


Table 10. Continued.

\begin{tabular}{|c|c|c|c|c|c|c|}
\hline $\begin{array}{l}\text { Trade Name } \\
\text { (Common Name) }\end{array}$ & $\begin{array}{l}\text { Rate } \\
\text { (product/acre) }\end{array}$ & $\begin{array}{l}\text { REI } \\
\text { (hours) }\end{array}$ & $\begin{array}{l}\text { Days to } \\
\text { Harvest }\end{array}$ & Insects & $\begin{array}{l}\text { MOA } \\
\text { Code }^{1}\end{array}$ & Notes \\
\hline $\begin{array}{l}\text { Deliver } \\
\text { (Bacillus thuringiensis } \\
\text { subspecies kurstaki) }\end{array}$ & $0.25-1.5 \mathrm{lb}$ & 4 & 0 & caterpillars & 11B2 & $\begin{array}{l}\text { Use higher rates for armyworms. } \\
\text { OMRI-listed². }\end{array}$ \\
\hline $\begin{array}{l}\text { Dimethoate } 4 \text { EC, } \\
2.67 \\
\text { (dimethoate) }\end{array}$ & $\begin{array}{l}\text { 4EC: } 0.5 \mathrm{pt} \\
2.67: 0.75 \mathrm{pt}\end{array}$ & 48 & 14 & $\begin{array}{l}\text { aphids, leafhoppers, } \\
\text { leafminers }\end{array}$ & 1B & \\
\hline \multirow[t]{2}{*}{$\begin{array}{l}\text { *Diazinon, * } 4 \text { EC, } \\
\text { *50 W } \\
\text { (diazinon) }\end{array}$} & $\begin{array}{l}\text { AG500, 4EC: } \\
0.5-1 \mathrm{pt} \\
\text { 50W: } 0.5-1 \mathrm{lb}\end{array}$ & 24 & 14 & aphids, leafminers & 1B & Limited to 5 applications. \\
\hline & $\begin{array}{l}\text { preplant - } \\
\text { AG500, 4EC: } 1- \\
4 \text { qts } \\
50 W: 2-8 \mathrm{lb}\end{array}$ & 24 & preplant & $\begin{array}{l}\text { cutworms, mole crickets, } \\
\text { wireworms }\end{array}$ & $1 \mathrm{~B}$ & See label. \\
\hline $\begin{array}{l}\text { DiPel DF } \\
\text { (Bacillus thuringiensis } \\
\text { subspecies kurstaki) }\end{array}$ & $0.5-2.0 \mathrm{lb}$ & 4 & 0 & caterpillars & 11B2 & $\begin{array}{l}\text { Treat when larvae are young. } \\
\text { Good coverage is essential. Can } \\
\text { be used in greenhouse. OMRI- } \\
\text { listed }^{2} \text {. }\end{array}$ \\
\hline $\begin{array}{l}\text { Entrust } \\
\text { (spinosad) }\end{array}$ & $0.5-3.0 \mathrm{oz}$ & 4 & 1 & $\begin{array}{l}\text { armyworms, cabbage } \\
\text { looper, leafminers, thrips }\end{array}$ & 5 & $\begin{array}{l}\text { Do not apply more than } 9 \text { oz per } \\
\text { acre per crop. See label for resis- } \\
\text { tance management recommenda- } \\
\text { tions. OMRI-listed². }\end{array}$ \\
\hline $\begin{array}{l}\text { Extinguish } \\
\text { ((S)-methoprene) }\end{array}$ & $1-1.5 \mathrm{lb}$ & 4 & 0 & fire ants & $7 \mathrm{~A}$ & $\begin{array}{l}\text { Slow-acting IGR (insect growth } \\
\text { regulator). Best applied early } \\
\text { spring and fall where crop will be } \\
\text { grown. Colonies will be reduced } \\
\text { after three weeks and eliminated } \\
\text { after } 8 \text { to } 10 \text { weeks. }\end{array}$ \\
\hline $\begin{array}{l}\text { Fulfill } \\
\text { (pymetrozine) }\end{array}$ & $2.75 \mathrm{oz}$ & 12 & 7 & $\begin{array}{l}\text { aphids, suppression of } \\
\text { whiteflies }\end{array}$ & $9 B$ & $\begin{array}{l}\text { Do not exceed } 5.5 \text { oz product per } \\
\text { acre per season. }\end{array}$ \\
\hline $\begin{array}{l}\text { Intrepid 2F } \\
\text { (methoxyfenozide) }\end{array}$ & $4-16 \mathrm{fl} \mathrm{oz}$ & 4 & 1 & $\begin{array}{l}\text { armyworms, cabbage } \\
\text { looper }\end{array}$ & 18 & $\begin{array}{l}\text { Do not apply more than } 64 \mathrm{fl} \mathrm{oz/} \\
\text { acre per season. }\end{array}$ \\
\hline $\begin{array}{l}\text { Javelin WG } \\
\text { (Bacillus thuringiensis } \\
\text { subspecies kurstaki) }\end{array}$ & $0.12-1.50 \mathrm{lb}$ & 4 & 0 & $\begin{array}{l}\text { most caterpillars, but } \\
\text { not Spodoptera species } \\
\text { (armyworms) }\end{array}$ & 11B2 & $\begin{array}{l}\text { Treat when larvae are young. } \\
\text { Thorough coverage is essential. } \\
\text { OMRI-listed?2. }^{2}\end{array}$ \\
\hline $\begin{array}{l}\text { *Lannate LV; *SP } \\
\text { (methomyl) }\end{array}$ & $\begin{array}{l}\text { LV: } \\
1.5-3.0 \mathrm{pt} \\
\text { SP: } \\
0.5-1.0 \mathrm{lb}\end{array}$ & 48 & 10 & beet armyworm & $1 \mathrm{~A}$ & \\
\hline $\begin{array}{l}{ }^{*} \text { Larvin } 3.2 \\
\text { (thiodicarb) }\end{array}$ & $16-30 \mathrm{fl} \mathrm{oz}$ & 48 & 14 & $\begin{array}{l}\text { armyworms, beet army- } \\
\text { worm, cabbage looper, } \\
\text { corn earworm, fall army- } \\
\text { worm, tomato fruitworm, } \\
\text { southern armyworm }\end{array}$ & $1 \mathrm{~A}$ & $\begin{array}{l}\text { Do not exceed } 1.5 \mathrm{lb} \text { active ingre- } \\
\text { dient per acre per season. }(60 \mathrm{fl} \\
\text { oz) }\end{array}$ \\
\hline $\begin{array}{l}\text { Lepinox WDG } \\
\text { (Bacillus thuringiensis } \\
\text { subspecies kurstaki) }\end{array}$ & $1.0-2.0 \mathrm{lb}$ & 12 & 0 & $\begin{array}{l}\text { most caterpillars, includ- } \\
\text { ing beet armyworm (see } \\
\text { label) }\end{array}$ & 11B2 & $\begin{array}{l}\text { Treat when larvae are small. } \\
\text { Thorough coverage is essential. }\end{array}$ \\
\hline $\begin{array}{l}\text { Malathion 8F } \\
\text { (malathion) }\end{array}$ & $2 \mathrm{pt}$ & 12 & 7 & aphids, leafhoppers, mites & $1 \mathrm{~B}$ & $\begin{array}{l}\text { Can be used in } \\
\text { greenhouse. }\end{array}$ \\
\hline $\begin{array}{l}\text { M-Pede } 49 \% \text { EC } \\
\text { Soap, insecticidal }\end{array}$ & $1-2 \%$ V/V & 12 & 0 & $\begin{array}{l}\text { aphids, leafhoppers, } \\
\text { mites, plant bugs, thrips, } \\
\text { whiteflies }\end{array}$ & -- & OMRI-listed². \\
\hline
\end{tabular}


Table 10. Continued.

\begin{tabular}{|c|c|c|c|c|c|c|}
\hline $\begin{array}{l}\text { Trade Name } \\
\text { (Common Name) }\end{array}$ & $\begin{array}{l}\text { Rate } \\
\text { (product/acre) }\end{array}$ & $\begin{array}{l}\text { REI } \\
\text { (hours) }\end{array}$ & $\begin{array}{l}\text { Days to } \\
\text { Harvest }\end{array}$ & Insects & $\begin{array}{l}\text { MOA } \\
\text { Code }^{1}\end{array}$ & Notes \\
\hline $\begin{array}{l}\text { Neemix } 4.5 \\
\text { (azadirachtin) }\end{array}$ & $4-16 \mathrm{fl} \mathrm{oz}$ & 12 & 0 & $\begin{array}{l}\text { aphids, armyworms, cab- } \\
\text { bage looper, cutworms, } \\
\text { leafminers, thrips, white- } \\
\text { flies }\end{array}$ & 26 & OMRI-listed?2. \\
\hline $\begin{array}{l}\text { Oberon 2SC } \\
\text { (spiromesifen) }\end{array}$ & $7.0-8.5 \mathrm{fl} \mathrm{oz}$ & 12 & 7 & whiteflies & 23 & $\begin{array}{l}\text { Maximum amount per crop: } 25.5 \\
\text { fl oz. No more than } 3 \text { applications }\end{array}$ \\
\hline $\begin{array}{l}\text { *Pounce } 3.2 \text { EC (per- } \\
\text { methrin) }\end{array}$ & $2-80 z$ & 12 & 1 & $\begin{array}{l}\text { aphids, armyworms, } \\
\text { beet armyworm, cabbage } \\
\text { looper, corn earworm, } \\
\text { cutworms, fall armyworm, } \\
\text { leafhoppers, leafminers }\end{array}$ & 3 & \\
\hline $\begin{array}{l}\text { *Proclaim } \\
\text { (emamectin benzoate) }\end{array}$ & $2.4-4.80 z$ & 48 & 7 & $\begin{array}{l}\text { beet armyworm, corn } \\
\text { earworm, fall armyworm, } \\
\text { loopers, tobacco bud- } \\
\text { worm, suppression of } \\
\text { leafminers }\end{array}$ & 6 & $\begin{array}{l}\text { Do not apply more than } 28.8 \text { oz/ } \\
\text { acre per season. }\end{array}$ \\
\hline $\begin{array}{l}\text { Provado 1.6F } \\
\text { (imidacloprid) }\end{array}$ & $3.80 z$ & 12 & 7 & $\begin{array}{l}\text { aphids, flea beetles, } \\
\text { whiteflies }\end{array}$ & $4 \mathrm{~A}$ & \\
\hline $\begin{array}{l}\text { Pyrellin EC } \\
\text { (pyrethrin + rotenone) }\end{array}$ & $1-2 \mathrm{pt}$ & 12 & $\begin{array}{l}12 \\
\text { hours }\end{array}$ & $\begin{array}{l}\text { aphids, flea beetles, leaf- } \\
\text { hoppers, leafminers, loop- } \\
\text { ers, Lygus bug, mites, } \\
\text { plant bugs, stink bugs, } \\
\text { thrips, whiteflies }\end{array}$ & 3 & \\
\hline $\begin{array}{l}\text { Sevin 80S, XLR, 4F } \\
\text { (carbaryl) }\end{array}$ & $\begin{array}{l}\text { 80S: } 0.63-2.5 \mathrm{lb} \\
\text { XLR, 4F: } 0.5- \\
2.0 \text { qt }\end{array}$ & 12 & 14 & $\begin{array}{l}\text { armyworms, corn ear- } \\
\text { worm, fall armyworm, flea } \\
\text { beetles, harlequin bugs, } \\
\text { leafhoppers, Lygus bug, } \\
\text { spittlebugs, stink bugs, } \\
\text { tarnished plant bug }\end{array}$ & $1 \mathrm{~A}$ & \\
\hline $\begin{array}{l}\text { SpinTor } 2 \text { SC } \\
\text { (spinosad) }\end{array}$ & $1.5-10 \mathrm{fl} \mathrm{oz}$ & 4 & 1 & $\begin{array}{l}\text { armyworms, cabbage } \\
\text { looper, leafminers } \\
\text { (Liriomyza spp.), thrips }\end{array}$ & 5 & $\begin{array}{l}\text { Do not apply more than } 29 \mathrm{fl} \mathrm{oz} \\
\text { per acre per crop. }\end{array}$ \\
\hline $\begin{array}{l}\text { *Telone C-35 (dichlo- } \\
\text { ropropene + chloro- } \\
\text { picrin) }\end{array}$ & See label & $\begin{array}{l}5 \text { days } \\
- \text { See } \\
\text { label }\end{array}$ & preplant & symphylans, wireworms & -- & $\begin{array}{l}\text { See supplemental label for use } \\
\text { restriction in south and central } \\
\text { Florida. }\end{array}$ \\
\hline \multicolumn{7}{|l|}{$\begin{array}{l}\text { *Telone II } \\
\text { (dichloropropene) }\end{array}$} \\
\hline $\begin{array}{l}\text { Trigard } \\
\text { (cryomazine) }\end{array}$ & $2.66 \mathrm{oz}$ & 12 & 7 & leafminers & 17 & $\begin{array}{l}\text { Do not apply more than } 6 \text { times } \\
\text { per crop. }\end{array}$ \\
\hline $\begin{array}{l}\text { Trilogy } \\
\text { (extract of neem oil) }\end{array}$ & $0.5-2.0 \% \mathrm{VN}$ & 4 & 0 & $\begin{array}{l}\text { aphids, mites, suppres- } \\
\text { sion of thrips and white- } \\
\text { flies }\end{array}$ & 26 & $\begin{array}{l}\text { Apply morning or evening to } \\
\text { reduce potential for leaf burn. } \\
\text { Toxic to bees exposed to direct } \\
\text { treatment. OMRI-listed?2. }\end{array}$ \\
\hline $\begin{array}{l}\text { Venom } \\
\text { (dinotefuran) }\end{array}$ & $\begin{array}{l}\text { foliar: } 1-3 \text { oz } \\
\text { soil: } 5-6 \text { oz }\end{array}$ & 12 & $\begin{array}{l}\text { foliar } \\
-7 \\
\text { soil } \\
-21\end{array}$ & $\begin{array}{l}\text { green peach aphid, } \\
\text { leafhoppers, leafminers, } \\
\text { potato aphid, whiteflies }\end{array}$ & $4 \mathrm{~A}$ & $\begin{array}{l}\text { Do not apply more than } 6 \text { oz per } \\
\text { acre per season (foliar) or12 oz } \\
\text { per acre per season (soil). Do not } \\
\text { use both methods of application. }\end{array}$ \\
\hline $\begin{array}{l}\text { Xentari DF } \\
\text { (Bacillus thuringiensis } \\
\text { subspecies aizawai) }\end{array}$ & $0.5-2.0 \mathrm{lb}$ & 4 & 0 & caterpillars & $11 \mathrm{~B} 1$ & $\begin{array}{l}\text { Treat when larvae are young. } \\
\text { Thorough coverage is essential. } \\
\text { May be used in the greenhouse. } \\
\text { Can be used in organic produc- } \\
\text { tion. }\end{array}$ \\
\hline
\end{tabular}


Table 10. Continued.

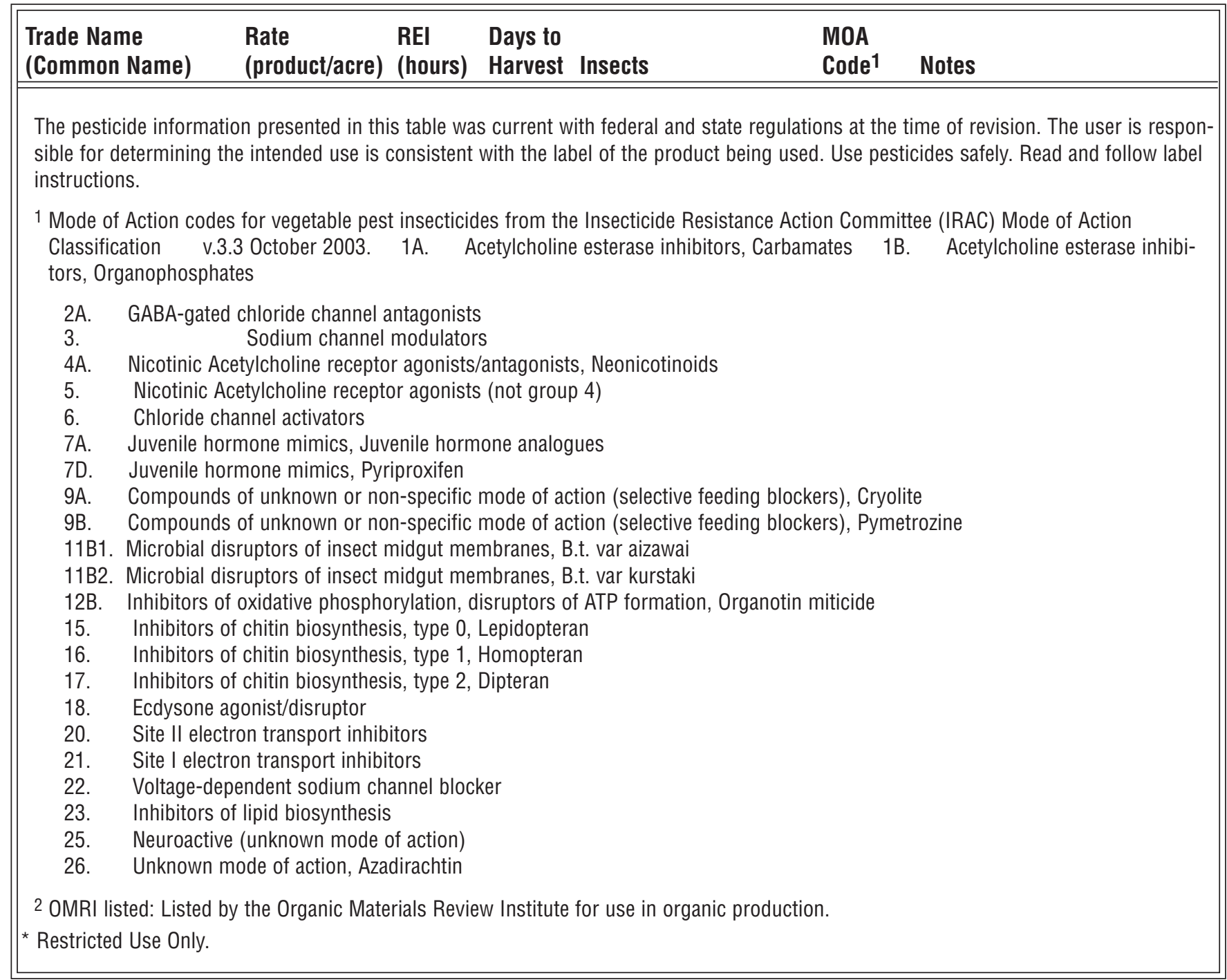

